\title{
OPTIMAL PARTIAL REGULARITY FOR NONLINEAR SUB-ELLIPTIC SYSTEMS RELATED TO HÖRMANDER'S VECTOR FIELDS
}

\author{
Jialin WANG and Dongni LIAO \\ (Received 21 July 2010 and revised 10 February 2011)
}

\begin{abstract}
This paper is concerned with partial regularity for weak solutions to nonlinear sub-elliptic systems related to Hörmander's vector fields. The method of $\mathcal{A}$-harmonic approximation introduced by Simon and developed by Duzaar and Grotowski is adapted to our context, and then a Caccioppoli-type inequality and partial regularity with optimal local Hölder exponent for gradients of weak solutions to the systems under super-quadratic natural growth conditions is established.
\end{abstract}

\section{Introduction and statements of main results}

Let $X_{1}, \ldots, X_{k}$ be a family of smooth vector fields defined in $\Omega \subset \mathbb{R}^{n}(n>k)$, i.e.,

$$
X_{i}=\sum_{j=1}^{n} b_{i j}(\xi) \frac{\partial}{\partial \xi_{j}}, \quad b_{i j}(\xi) \in C^{\infty}(\Omega), i=1,2, \ldots, k .
$$

Hörmander in [18] showed that if smooth vector fields $X_{1}, \ldots, X_{k}$ satisfy the finite rank condition (rank $\operatorname{Lie}\left[X_{1}, \ldots, X_{k}\right]=n$ at every point $\xi \in \mathbb{R}^{n}$ ), then the square sum operator $L=\sum_{i=1}^{k} X_{i}^{*} X_{i}$ is hypoelliptic, where $X_{i}^{*}$ is the formal adjoint of $X_{i}$. For example, in typical cases of Heisenberg groups, groups of Heisenberg type and even general Carnot groups, their Lie algebras have a family of smooth vector fields satisfying Hörmander's rank condition, so the sub-elliptic Laplacian consisting of those vector fields is hypoelliptic. Since the appearance of Hörmander's work [18], the study of properties for weak solutions to linear or nonlinear equations and systems arising from Hörmander's vector fields has attracted extensive attention. Although some new and serious difficulties arose due to noncommutativity of vector fields in the study of regularity theory, many significant results for regularity of weak solutions to these equations were established; see $[\mathbf{1 , ~ 2 , ~ 5 , ~ 1 6 - 2 1 , ~ 2 6 , ~}$ 28-30] and references therein.

Especially, Xu and Zuily in [30] investigated the Hölder continuity for the gradient of weak solutions to the quasi-linear system

$$
\sum_{i, j=1}^{k} X_{j}^{*}\left(a^{i j}(\xi, u) X_{i} u^{\alpha}\right)=f^{\alpha}(\xi, u, X u), \quad \alpha=1, \ldots, N
$$

2000 Mathematics Subject Classification: Primary 35H20, 35B65.

Keywords: $\mathcal{A}$-harmonic approximation technique; partial regularity; nonlinear sub-elliptic systems; Hörmander's vector fields; super-quadratic natural growth condition. 
for Hörmander's vector fields $X_{1}, \ldots, X_{k}$, where $a^{i j}(\xi, u)$ is a positive definite symmetry matrix, and $f^{\alpha}$ satisfies quadratic growth conditions. Later, Giuseppe and Maria in [16] showed the local Morrey regularity for the gradient of weak solutions to the following linear system involving Hörmander's vector fields:

$$
-X_{j}^{*}\left(a_{\alpha \beta}^{i j}(\xi) X_{i} u^{\beta}\right)=g_{i}-X_{j}^{*} f_{i}^{\alpha}, \quad \alpha=1, \ldots, N
$$

with suitable assumptions of $g_{i}$ and $f_{i}^{\alpha}$, where $a_{\alpha \beta}^{i j}(\xi)$ are bounded functions and belong to the vanishing mean oscillation space.

However, in the Euclidean space $\mathbb{R}^{n}$, many interesting regularity results for more general nonlinear elliptic systems have been established, based on the latest method called $\mathcal{A}$-harmonic approximation. For example, Duzaar and Grotowski in [12] treated general nonlinear systems under quadratic growth conditions, and then both cases of superquadratic natural growth conditions and super-quadratic controllable growth conditions were considered by Chen and Tan in [6] and [7], respectively. We also note that the degenerate $p$-Laplacian version of the method has been obtained by Duzaar and Mingione in [10], and applied to the partial regularity by the same authors in [11].

The purpose of this paper is to establish the Hölder continuity for the gradient of weak solutions to more general sub-elliptic systems structured by Hörmander's vector fields under super-quadratic natural growth conditions.

Precisely, let $\Omega \subset \mathbb{R}^{n}$ be a bounded, open and path-connected domain, and consider the nonlinear sub-elliptic system

$$
\sum_{i=1}^{k} X_{i}^{*} A_{i}^{\alpha}(\xi, u, X u)=B^{\alpha}(\xi, u, X u), \quad(\xi, u, X u) \in \Omega \times \mathbb{R}^{N} \times \mathbb{R}^{k N}, \alpha=1, \ldots, N
$$

under the following super-quadratic structure conditions (H1)-(H3) and (N).

(H1) $A_{i}^{\alpha}(\xi, u, p)$ are differentiable functions with respect to $p$, with bounded and continuous derivatives, that is, there exists a constant $C$ such that

$$
\left|A_{i, p_{\beta}^{j}}^{\alpha}(\xi, u, p)\right| \leq C\left(1+|p|^{2}\right)^{(m-2) / 2}, \quad(\xi, u, p) \in \Omega \times \mathbb{R}^{N} \times \mathbb{R}^{k N}, m \geq 2,
$$

where we denote $A_{i, p_{\beta}^{j}}^{\alpha}(\cdot)=\partial A_{i}^{\alpha}(\cdot) / \partial p_{\beta}^{j}$.

(H2) $A_{i}^{\alpha}(\xi, u, p)$ satisfies the ellipticity condition

$$
A_{i, p_{\beta}^{j}}^{\alpha}(\xi, u, p) \eta_{i}^{\alpha} \eta_{j}^{\beta} \geq \lambda\left(1+|p|^{2}\right)^{(m-2) / 2}|\eta|^{2}, \quad \forall \eta \in \mathbb{R}^{k N},
$$

where $\lambda$ is a positive constant.

(H3) $A_{i}^{\alpha}(\xi, u, p)$ is Hölder continuous with exponent $\gamma \in(0,1)$ in the first and second variables, respectively, i.e.,

$$
\left|A_{i}^{\alpha}(\xi, u, p)-A_{i}^{\alpha}(\tilde{\xi}, \tilde{u}, p)\right| \leq K(|u|)\left(\varrho_{\mathrm{cc}}^{m}(\xi, \tilde{\xi})+|u-\tilde{u}|^{m}\right)^{\gamma / m}\left(1+|p|^{2}\right)^{(m-1) / 2},
$$

where $K(\cdot):[0, \infty) \rightarrow[0, \infty)$ is monotonously non-decreasing, and $\varrho_{\mathrm{cc}}(\xi, \tilde{\xi})$ is the so-called Carnot-Carathéodory metric between $\xi$ and $\tilde{\xi}$ (see (2.1) below). Without loss of generality, it is convenient to take $K(\cdot) \geq 1$. 
(N) (Natural growth condition) For $|u| \leq M=\sup _{\Omega}|u|, B^{\alpha}$ satisfies

$$
\left|B^{\alpha}(\xi, u, p)\right| \leq a|p|^{m}+b
$$

where $a$ (possibly depending on $M>0$ ) and $b$ are positive constants.

Next, we give some explanation about these assumptions. Note that (H1) infers

$$
\left|A_{i}^{\alpha}(\xi, u, p)-A_{i}^{\alpha}(\xi, u, \tilde{p})\right| \leq C\left(1+|p|^{2}+|\tilde{p}|^{2}\right)^{(m-2) / 2}|p-\tilde{p}| .
$$

Moreover, there exists a bounded and continuously non-negative function $\omega(s, t):[0, \infty) \times$ $[0, \infty) \rightarrow[0, \infty)$, where $\omega(s, 0)=0$ for all $s$, and $\omega(s, t)$ is monotonously non-decreasing in $s$ for fixed $t ; \omega(s, t)$ is monotonously non-decreasing and concave in $t$ for fixed $s$, such that for all $\xi \in \Omega, u \in \mathbb{R}^{N}$ and $p, \tilde{p} \in \mathbb{R}^{k N}$,

$$
\left|A_{i, p_{\beta}^{j}}^{\alpha}(\xi, u, p)-A_{i, p_{\beta}^{j}}^{\alpha}(\xi, u, \tilde{p})\right| \leq C\left(1+|p|^{2}+|\tilde{p}|^{2}\right)^{(m-2) / 2} \omega(|p|,|p-\tilde{p}|) .
$$

From (H2), we have

$$
\left(A_{i}^{\alpha}(\xi, u, p)-A_{i}^{\alpha}(\xi, u, \tilde{p})\right)(p-\tilde{p}) \geq \lambda^{\prime}\left(|p-\tilde{p}|^{2}+|p-\tilde{p}|^{m}\right),
$$

with a positive constant $\lambda^{\prime}$.

In fact, it is easy to follow that

$$
\begin{aligned}
& \left(A_{i}^{\alpha}(\xi, u, p)-A_{i}^{\alpha}(\xi, u, \tilde{p})\right)\left(p_{i}^{\alpha}-\tilde{p}_{i}^{\alpha}\right) \\
& \quad=\int_{0}^{1} \frac{d}{d t} A_{i}^{\alpha}(\xi, u, t p+(1-t) \tilde{p}) d t\left(p_{i}^{\alpha}-\tilde{p}_{i}^{\alpha}\right) \\
& \quad=\int_{0}^{1} \frac{\partial}{\partial p_{j}^{\beta}} A_{i}^{\alpha}(\xi, u, t p+(1-t) \tilde{p})\left(p_{j}^{\beta}-\tilde{p}_{j}^{\beta}\right)\left(p_{i}^{\alpha}-\tilde{p}_{i}^{\alpha}\right) d t \\
& \quad \geq \lambda \int_{0}^{1}\left(1+|t p+(1-t) \tilde{p}|^{2}\right)^{(m-2) / 2}|p-\tilde{p}|^{2} d t .
\end{aligned}
$$

Using the following inequality from Lemma 2.1 in [15]

$$
2^{-2(1+\delta)} \leq \frac{\int_{0}^{1}\left(1+|t p+(1-t) \tilde{p}|^{2}\right)^{\delta / 2} d t}{\left(1+|p|^{2}+|\tilde{p}|^{2}\right)^{\delta / 2}} \leq 2^{\delta / 2}, \quad \forall \delta \geq 0
$$

gives (1.8).

In the present paper, we will apply the method of $\mathcal{A}$-harmonic approximation adapting to the setting of Hörmander vector fields to study partial regularity for the system (1.1). The general steps are as follows.

(1) A Caccioppoli-type inequality is established for weak solutions of (1.1).

(2) The inequality and the $\mathcal{A}$-harmonic approximation technique lead to decay estimates for the following function:

$$
\Phi\left(\xi_{0}, \rho, p_{0}\right)=\left|B_{\rho}\left(\xi_{0}\right)\right|^{-1} \int_{B_{\rho}\left(\xi_{0}\right)}\left[\left|X u-p_{0}\right|^{2}+\left|X u-p_{0}\right|^{m}\right] d \xi .
$$

(3) Partial regularity for gradients of weak solutions is obtained by the standard iteration. 
As in the Euclidean space, when coefficients $A_{i}^{\alpha}$ are Hölder continuous with exponent $\gamma$, one hopes to see that the gradients of weak solutions to systems have Hölder regularity with the same exponent. However, a preliminary experiment with the classical direct technique can only get the Hölder exponent less than $\gamma$. Fortunately, based on the work in this paper, we obtain the desired optimal Hölder exponent $\gamma$ for weak solutions.

The main result in this paper is the following theorem.

THEOREM 1.1. Assume that coefficients $A_{i}^{\alpha}$ and $B^{\alpha}$ satisfy conditions $(H 1)-(H 3)$ and $(N)$, and let $u \in H W^{1, m}\left(\Omega, \mathbb{R}^{N}\right) \cap L^{\infty}\left(\Omega, \mathbb{R}^{N}\right)$ be a weak solution to the system (1.1) with $\Omega \subset \mathbb{R}^{n}$ and $2 a M<\lambda^{\prime}$, i.e.,

$$
\int_{\Omega} A_{i}^{\alpha}(\xi, u, X u) X_{i} \varphi^{\alpha} d \xi=\int_{\Omega} B^{\alpha}(\xi, u, X u) \varphi^{\alpha} d \xi \quad \forall \varphi \in C_{0}^{\infty}\left(\Omega, \mathbb{R}^{N}\right) .
$$

Then there exists an open subset $\Omega_{0} \subset \Omega$ such that $u \in \Gamma^{1, \gamma}\left(\Omega_{0}, \mathbb{R}^{N}\right)$, with $\gamma$ in (1.4). Furthermore, $\Omega \backslash \Omega_{0}=\Sigma_{1} \cup \Sigma_{2}$ and meas $\left(\Omega \backslash \Omega_{0}\right)=0$, where

$$
\begin{aligned}
& \Sigma_{1}=\left\{\xi_{0} \in \Omega: \lim _{r \rightarrow 0^{+}} \sup \left(\left|u_{\xi_{0}, r}\right|+\left|(X u)_{\xi_{0}, r}\right|\right)=\infty\right\}, \\
& \Sigma_{2}=\left\{\xi_{0} \in \Omega: \lim _{r \rightarrow 0^{+}} \inf \left|B_{r}\left(\xi_{0}\right)\right|^{-1} \int_{B_{r}\left(\xi_{0}\right)}\left|X u-(X u)_{\xi_{0}, r}\right|^{m} d \xi>0\right\} .
\end{aligned}
$$

The condition $2 a M<\lambda^{\prime}$ in Theorem 1.1 is necessary. Whether we may assume $a M<\lambda^{\prime}$ instead of the condition is still an open issue. The following result shows that we can slightly change (1.5).

THEOREM 1.2. If the natural growth condition (1.5) is replaced by $\left|B^{\alpha}(\xi, u, p)\right| \leq$ $a|p|^{m-\epsilon}+b$ with $\epsilon>0$ and the requirement $2 a M<\lambda^{\prime}$ in Theorem 1.1 is cancelled, then the conclusion of Theorem 1.1 is still valid.

To the best of our knowledge, in the Heisenberg group, $\Gamma^{0, \gamma}$ regularity is valid for $1<p<\infty$ and was proved by several people during the 1990s [5, 21, 29], but when turning to $\Gamma^{1, \gamma}$ regularity, it is worth pointing out that the remarkable contribution of Hölder continuity for the gradient of weak solutions to sub-elliptic $p$-Laplacian equations is due to Capogna [3, 4], Marchi [22, 23], Domokos [8, 9], Manfredi and Mingione [24] and Mingione et al [25], but the exponent $p$ should be near 2, and the limitation $2 \leq p<4$ appears in the most recent of the cited works. Recently, Garofalo in [14] obtained the $\Gamma^{1, \gamma}$ regularity of weak solutions which possess some special symmetries for $2 \leq p$. When turning to partial continuity of weak solutions, our results show that the $\Gamma^{1, \gamma}$ continuity is also valid for exponent $2 \leq p$.

Compared with the usual gradient vector fields $\left\{X_{i}=\partial / \partial x_{i}, i=1, \ldots, n\right\}$ on the Euclidean case $\mathbb{R}^{n}$, there exist two main difficulties in our setting: the lack of commutation and the lack of homogeneity of the vector fields. The first difficulty does not allow us to differentiate the equation, and the second difficulty does not permit us to use scaling. Also, Hörmander's vector fields have no explicit expressions like basic vector fields in Carnot groups, and we meet an obstacle when trying to establish Caccioppoli-type inequalities. Fortunately, a result on the expressions of vector fields by Xu and Zuily in [30] plays a crucial role in proving the inequality. 
The structure of this paper is as follows. In Section 2, we collect some basic notion and facts related to Hörmander's vector fields, involving the Carnot-Carathéodory metric, the Sobolev-type embedding theorem, the Poincaré-type inequality and several relative function spaces. In Section 3, an $\mathcal{A}$-harmonic approximation lemma is given and a conclusion for constant systems obtained by Xu and Zuily [30] is generalized. Then we show a Caccioppolitype inequality for weak solutions to the system (1.10). Section 4 is devoted to the proof of Theorems 1.1 and 1.2, and the process of the proof is split into four steps. An estimate on weak solutions is established in the first step. The second step begins with proving three 'small conditions'(see (4.10)-(4.12) below), and then obtaining the estimate for the function $\Phi\left(\xi_{0}, \rho, p_{0}\right)$ in (1.9). The third step is to show that we can iterate the statement in the second step. A decay estimate

$$
\Phi\left(\xi_{0}, \rho, p_{0}\right) \leq C \rho^{2 \gamma}
$$

is obtained in the last step, where $\gamma$ is the same as (1.4). Then we can infer the conclusion of Theorem 1.1 by employing the Campanato-type lemma by Capogna [4]. The proof of Theorem 1.2 is very similar to that of Theorem 1.1 and the key point is to find a suitable estimate for a new term (see (4.37) below).

\section{Basic facts and notion}

Assume that $\Omega \subset \mathbb{R}^{n}$ is a bounded, open and path-connected domain and that $\left\{X_{i}\right\}_{i=1}^{k}(k<n)$ are a family of $C^{\infty}$ real valued vector fields defined in a neighborhood of the closure $\bar{\Omega}$ of $\Omega$. For a multi-index $\alpha=\left(i_{1}, \ldots, i_{k}\right)$, we denote by $X_{\alpha}$ the commutator

$$
\left[X_{i_{1}},\left[X_{i_{2}}, \ldots,\left[X_{i_{k-1}}, X_{i_{k}}\right]\right]\right]
$$

of length $l=|\alpha|$. Throughout the paper, we suppose that the vector fields satisfy Hörmander's condition: there exist some positive integers $s$ such that $\left\{X_{\alpha}\right\}_{|\alpha| \leq s}$ span the tangent space of $\mathbb{R}^{n}$ at each point of $\Omega$, that is, $\operatorname{rank} \operatorname{Lie}\left[X_{1}, \ldots, X_{k}\right] \equiv n$.

Rothschild and Stein in [27] exhibited a lifting and approximation technique which implies that the original vector fields $\left\{X_{1}, \ldots, X_{k}\right\}$ can be lifted by adding extra variables to free vector fields $\left\{\widetilde{X}_{1}, \ldots, \widetilde{X}_{k}\right\}$ on $\Omega \times T \subset \mathbb{R}^{n} \times \mathbb{R}^{d-n}$ with $T$ being the unit ball in $\mathbb{R}^{d-n}$, and

$$
\tilde{X}_{i}=X_{i}+\sum_{l=1}^{d-n} \lambda_{i l}(\xi, t) \frac{\partial}{\partial t_{l}} .
$$

Note that we have $X_{i} u(\xi)=\tilde{X}_{i} u(\xi)$ for $u=u(\xi)$. So, if $u(\xi)$ is a solution of (1.1), then it also satisfies the system

$$
\sum_{i=1}^{k} \tilde{X}_{i}^{*} A_{i}^{\alpha}(\xi, u, \widetilde{X} u)=B^{\alpha}(\xi, u, \tilde{X} u), \quad \alpha=1, \ldots, N,
$$

where $\left\{\tilde{X}_{i}\right\}$ is a family of lifted vector fields of $\left\{X_{i}\right\}$ (see [27]). Without loss of generality, we may assume that $\left\{X_{1}, \ldots, X_{k}\right\}$ are free up to order $s$.

Now, we introduce a metric in the following way. Assume that admissible path $\gamma$ is a Lipschitz curve $\gamma:[0, b] \mapsto \Omega$, and there exist $c_{i}(t), 0 \leq t \leq b, i=1, \ldots, k$, satisfying

$$
\sum_{i=1}^{k} c_{i}(t)^{2} \leq 1 \quad \text { and } \quad \gamma^{\prime}(t)=\sum_{i=1}^{k} c_{i}(t) X_{i}(\gamma(t))
$$


for a.e. $t \in[0, b]$. Then a Carnot-Carathéodory (abbreviated to $\mathrm{C}-\mathrm{C}$ ) metric on $\Omega$ associated to $\left\{X_{i}\right\}_{i=1}^{k}$ is defined by

$$
\varrho_{\mathrm{cc}}(\xi, \eta)=\min \{b \geq 0: \exists \gamma:[0, b] \mapsto \Omega \text {, s.t. } \gamma(0)=\xi, \gamma(b)=\eta\} \text {. }
$$

From (2.1), we can define the $\mathrm{C}-\mathrm{C}$ ball and the $\mathrm{C}-\mathrm{C}$ sphere as

$$
B_{r}(\xi)=\left\{\eta: \varrho_{\mathrm{cc}}(\xi, \eta)<r\right\}, \quad \partial B_{r}(\xi)=\left\{\eta: \varrho_{\mathrm{cc}}(\xi, \eta)=r\right\},
$$

respectively. A fundamental doubling property of the Lebesgue measure with respect to the C-C metric balls was shown by Nagel et al in [26], namely: given a bounded set $\Omega \subset \mathbb{R}^{n}$, there are positive constants $C$ and $R_{0}$, such that

$$
\left|B_{2 R}(\xi)\right| \leq C\left|B_{R}(\xi)\right|, \quad \text { for } \xi \in \Omega \text { and } 0<R<R_{0} .
$$

Let $Q=\log _{2} C$, then the number $Q$ acts as a dimension and is therefore called the local homogeneous dimension relative to $\Omega$ and to $\left\{X_{i}\right\}_{i=1}^{k}$. When $\left\{X_{i}=\partial / \partial x_{i}, i=1, \ldots, n\right\}$, one has $C=2^{n}$ and $Q=n$. Furthermore,

$$
\left|B_{R}(\xi)\right|=\omega_{X}(\xi) R^{Q},
$$

where $\omega_{X}(\xi)$ is positively bounded and depends only on the center $\xi$.

The following result from [30] is crucial for our discussion.

LEMMA 2.1. Let $P=\sum_{i, m=1}^{k} X_{i}\left(a_{i m} X_{m}\right)$ be a second-order differential operator with $a_{i m}$ being functions and denote by $A$ the matrix $\left(a_{i m}\right)_{1 \leq i, m \leq k}$. For any $\xi_{0} \in \Omega$, one can find coordinates in a neighborhood $V$ of $\xi_{0}$ and a matrix $T(\xi) \in \mathrm{GL}(k, \mathbb{R})$ which is $C^{\infty}$ in $V$ such that if we set $\left(Y_{1}, \ldots, Y_{k}\right)=T(\xi)\left(X_{1}, \ldots, X_{k}\right)$, then:

$$
Y_{i}=\frac{\partial}{\partial \xi_{i}}+\sum_{l=k+1}^{n} g_{i l} \frac{\partial}{\partial \xi_{l}}, \quad i=1, \ldots, k,
$$

(2) the set $\left(Y_{1}, \ldots, Y_{k}\right)$ satisfies Hörmander's condition of order $s$ and is free up to the order $s$ in $V$;

$$
P=\sum_{i, m=1}^{k} Y_{i}\left(b_{i m} Y_{m}\right),
$$

where $\left(b_{i m}\right)=C^{T}(\xi) A C(\xi)$ with $C(\xi)$ an invertible matrix with $C^{\infty}$ entries in $V$.

Because of the result, we always assume that Hörmander's vector fields $X_{1}, \ldots, X_{k}$ have the form of $Y_{1}, \ldots, Y_{k}$ in (2.3).

Definition 2.2. Let $\Omega \subset \mathbb{R}^{n}$ be an open set and denote by

$$
H W^{1, m}(\Omega)=\left\{u \in L^{m}(\Omega) \mid X_{i} u \in L^{m}(\Omega), i=1, \ldots, k\right\}
$$

the Sobolev space. Then $H W^{1, m}(\Omega)$ is a Banach space under the norm

$$
\|u\|_{H W^{1, m}(\Omega)}=\|u\|_{L^{m}(\Omega)}+\sum_{i=1}^{k}\left\|X_{i} u\right\|_{L^{m}(\Omega)} .
$$

The local Sobolev space is defined by

$$
H W_{\mathrm{loc}}^{1, m}(\Omega)=\left\{u \mid \eta u \in H W^{1, m}(\Omega), \eta \in C_{0}^{\infty}(\Omega)\right\} .
$$


Jerison [19] showed a Poincaré-type inequality related to Hörmander's vector fields:

$$
\int_{B_{\rho}\left(\xi_{0}\right)}\left|u(\xi)-u_{\xi_{0}, \rho}\right|^{m} d \xi \leq C_{P} \rho^{m} \int_{B_{\rho}\left(\xi_{0}\right)}|X u|^{m} d \xi, \quad u \in H W^{1, m}\left(B_{\rho}\left(\xi_{0}\right), \mathbb{R}^{N}\right),
$$

where $u_{\xi_{0}, \rho}=\left|B_{\rho}\left(\xi_{0}\right)\right|^{-1} \int_{B_{\rho}\left(\xi_{0}\right)} u(\xi) d \xi$, and $C_{P}$ depends only on $m(m \geq 1)$ and $Q$. Without loss of generality, we may assume $C_{P}>1$.

Denote by $\operatorname{Bil}\left(\mathbb{R}^{k N}\right)$ the collection of bi-linear forms defined in $\mathbb{R}^{k N}$, and suppose $\mathcal{A} \in \operatorname{Bil}\left(\mathbb{R}^{k N}\right)$. We say that a function $h \in H W^{1, m}\left(\Omega, \mathbb{R}^{N}\right)$ is $\mathcal{A}$-harmonic, if $h$ satisfies

$$
\int_{\Omega} \mathcal{A}(X h, X \varphi) d \xi=0, \quad \forall \varphi \in C_{0}^{1}\left(\Omega, \mathbb{R}^{N}\right) .
$$

Definition 2.3. Let $\gamma \in(0,1)$ and denote

$$
\Gamma^{1, \gamma}(\Omega)=\left\{u \in L^{\infty}(\Omega) \mid X_{i} u \in \Gamma^{\gamma}(\Omega), i=1, \ldots, k\right\},
$$

where

$$
\Gamma^{\gamma}(\Omega)=\left\{u \in L^{\infty}(\Omega) \mid[u]_{0, \gamma: \Omega}=\sup _{\xi, \tilde{\xi} \in \Omega, \xi \neq \tilde{\xi}} \frac{|u(\xi)-u(\tilde{\xi})|}{\varrho_{\mathrm{cc}}(\xi, \tilde{\xi})^{\gamma}}<\infty\right\} .
$$

We say that $\Gamma^{1, \gamma}(\Omega)$ is a Folland-Stein space with the norm

$$
\|u\|_{\Gamma^{1, \gamma}(\Omega)}=\|u\|_{L^{\infty}(\Omega)}+\|X u\|_{L^{\infty}(\Omega)}+[u]_{0, \gamma: \Omega}+[X u]_{0, \gamma: \Omega} .
$$

Garofalo and Nhieu [17] proved a Sobolev-type embedding theorem for Hörmander's vector fields.

Lemma 2.4. Let $1 \leq m<Q$ with $Q$ being the homogeneous dimension. Then for every $u \in H W_{0}^{1, m}\left(B_{R}\left(\xi_{0}\right)\right)$, there exist positive constants $C$ and $R_{0}$, such that

$$
\left(\left|B_{R}\left(\xi_{0}\right)\right|^{-1} \int_{B_{R}\left(\xi_{0}\right)}|u|^{\kappa m} d \xi\right)^{1 / \kappa m} \leq C R\left(\left|B_{R}\left(\xi_{0}\right)\right|^{-1} \int_{B_{R}\left(\xi_{0}\right)}|X u|^{m} d \xi\right)^{1 / m},
$$

provided that $\xi_{0} \in \Omega, R \leq R_{0}$, where $1 \leq \kappa \leq Q /(Q-m)$.

Definition 2.5. Let $\Omega \subset \mathbb{R}^{n}, 1 \leq m<\infty, \mu \geq 0$, and denote

$$
\mathcal{L}^{m, \mu}(\Omega)=\left\{\left.u|| u\right|_{L^{m, \mu}(\Omega)}=\sup _{\xi \in \Omega, \rho<\operatorname{diam} \Omega} \rho^{-\mu} \int_{B_{\rho}(\xi) \cap \Omega}|u(\zeta)-u \xi \xi|^{m} d \zeta<\infty\right\} .
$$

Then $\mathcal{L}^{m, \mu}(\Omega)$ is said to be a Campanato space with the norm

$$
\|u\|_{\mathcal{L}^{m, \mu}(\Omega)}=\|u\|_{L^{m}(\Omega)}+|u|_{L^{m, \mu}(\Omega)} .
$$

We recall that an open set $\Omega \subset \mathbb{R}^{n}$ has the $A$-property if and only if there exists $A>0$ such that for every $\xi \in \Omega$ and $\rho>0$,

$$
\left|\Omega \cap B_{\rho}(\xi)\right| \geq A\left|B_{\rho}(\xi)\right| .
$$

Capogna in [4] pointed out that any gauge ball $B_{r}(\xi)$ fits the $A$-property and proved a Campanato-type lemma for Hörmander's vector fields. 
LeMmA 2.6. Let $m \geq 1, r>0$ and $Q<\mu \leq Q+m$, then

$$
\mathcal{L}^{m, \mu}\left(B_{\rho}(\xi)\right) \subset \Gamma^{\gamma}\left(B_{\rho}(\xi)\right), \quad \gamma=\frac{\mu-Q}{m} .
$$

It is useful to write the following.

Lemma 2.7. (Lyapunov's inequality) Suppose $0<m, q<\infty, f \in L^{m}(\Omega) \cap L^{q}(\Omega)$. Then for any $r$ with $m<r<q$, we have $f \in L^{r}(\Omega)$ and

$$
\|f\|_{r} \leq\|f\|_{m}^{1-\alpha}\|f\|_{q}^{\alpha},
$$

where $0<\alpha<1$ with $1 / r=(1-\alpha) / m+\alpha / q$.

The conclusion can be obtained by Hölder's inequality.

In this paper, we denote by $C$ a general constant, which may vary from line to line.

\section{Caccioppoli-type inequality}

Similar to [12], one can establish the following $\mathcal{A}$-harmonic approximation lemma for vector fields.

Lemma 3.1. Consider fixed positive $\lambda$ and $L$, and $k, N \in \mathbb{N}$ with $k \geq 2$. Assume that

$$
\mathcal{A}(v, v) \geq \lambda|v|^{2}, \quad \mathcal{A}(v, \bar{v}) \leq L|v \| \bar{v}|, \quad v, \bar{v} \in \mathbb{R}^{k N}, \mathcal{A} \in \operatorname{Bil}\left(\mathbb{R}^{k N}\right)
$$

and

$$
\left|B_{\rho}\left(\xi_{0}\right)\right|^{-1} \int_{B_{\rho}\left(\xi_{0}\right)}|X g|^{2} d \xi \leq 1, \quad g \in H W^{1, m}\left(B_{\rho}\left(\xi_{0}\right), \mathbb{R}^{N}\right) .
$$

Then for any given $\varepsilon>0$, there exists $\delta=\delta(k, N, \lambda, \varepsilon) \in(0,1]$ such that if

$$
\left.|| B_{\rho}\left(\xi_{0}\right)\right|^{-1} \int_{B_{\rho}\left(\xi_{0}\right)} \mathcal{A}(X g, X \varphi) d \xi\left|\leq \delta \sup _{B_{\rho}\left(\xi_{0}\right)}\right| X \varphi \mid, \quad \forall \varphi \in C_{0}^{1}\left(B_{\rho}\left(\xi_{0}\right), \mathbb{R}^{N}\right)
$$

is satisfied, then

$$
\left|B_{\rho}\left(\xi_{0}\right)\right|^{-1} \rho^{-2} \int_{B_{\rho}\left(\xi_{0}\right)}|h-g|^{2} d \xi \leq \epsilon
$$

holds, where $h$ is an $\mathcal{A}$-harmonic function in $H W^{1, m}\left(B_{\rho}\left(\xi_{0}\right), \mathbb{R}^{N}\right)$ satisfying

$$
\left|B_{\rho}\left(\xi_{0}\right)\right|^{-1} \int_{B_{\rho}\left(\xi_{0}\right)}|X h|^{2} d \xi \leq 1 .
$$

Proof. We assume the conclusion to be false, and then for some $\varepsilon>0$ we can find $\left\{\mathcal{A}_{k}\right\}$ each fulfilling (3.1), and $\left\{g_{k}\right\}$ satisfying (3.2) and (3.3) with $\delta_{k}=1 / k$, but failing to obey (3.4); that is,

$$
\left|B_{\rho}\left(\xi_{0}\right)\right|^{-1} \rho^{-2} \int_{B_{\rho}\left(\xi_{0}\right)}\left|h_{k}-g_{k}\right|^{2} d \xi \geq \varepsilon \quad \text { for all } \mathcal{A}_{k} \text {-harmonic functions. }
$$

Without loss of generality, we may assume $\left|B_{\rho}\left(\xi_{0}\right)\right|^{-1} \int_{B_{\rho}\left(\xi_{0}\right)} g_{k} d \xi=0$ (otherwise consider $\left.g_{k}-\left|B_{\rho}\left(\xi_{0}\right)\right|^{-1} \int_{B_{\rho}\left(\xi_{0}\right)} g_{k} d \xi\right)$. By the compact embedding of $H W^{1, p}$ into $L^{p}$, we 
find subsequences of $g_{k}$ (also denoted by $\left\{g_{k}\right\}$ ), $g$ and $\mathcal{A}$ such that $g_{k} \rightarrow g$ weakly in $H W^{1,2}$, $g_{k} \rightarrow g$ strongly in $L^{2}, \mathcal{A}_{k} \rightarrow \mathcal{A}$ and $\left|B_{\rho}\left(\xi_{0}\right)\right|^{-1} \int_{B_{\rho}\left(\xi_{0}\right)}|X g| d \xi \leq 1$.

Next, we further find that $g$ is $A$-harmonic by using the weak $H W^{1,2}$ convergence of $g_{k}$ to $g$ and $\mathcal{A}_{k}$-harmonicity of the $g_{k}$ and the convergence of the $\mathcal{A}_{k}$ :

$$
\begin{aligned}
\left|B_{\rho}\left(\xi_{0}\right)\right|^{-1} \int_{B_{\rho}\left(\xi_{0}\right)} \mathcal{A}(X g, X \varphi) d \xi= & \left|B_{\rho}\left(\xi_{0}\right)\right|^{-1} \int_{B_{\rho}\left(\xi_{0}\right)} \mathcal{A}\left(X\left(g-g_{k}\right), X \varphi\right) d \xi \\
& +\left|B_{\rho}\left(\xi_{0}\right)\right|^{-1} \int_{B_{\rho}\left(\xi_{0}\right)}\left(\mathcal{A}-\mathcal{A}_{k}\right)\left(X g_{k}, X \varphi\right) d \xi \\
& +\left|B_{\rho}\left(\xi_{0}\right)\right|^{-1} \int_{B_{\rho}\left(\xi_{0}\right)} \mathcal{A}_{k}\left(X g_{k}, X \varphi\right) d \xi \rightarrow 0 \quad(k \rightarrow \infty) .
\end{aligned}
$$

We now consider the Dirichlet problem given by

$$
\begin{gathered}
\left|B_{\rho}\left(\xi_{0}\right)\right|^{-1} \int_{B_{\rho}\left(\xi_{0}\right)} A_{k}(X w, X \varphi) d \xi=0, \\
\forall \varphi \in C_{0}^{1}\left(B_{\rho}\left(\xi_{0}\right), \mathbb{R}^{N}\right), w-g \in H W_{0}^{1,2}\left(B_{\rho}\left(\xi_{0}\right), \mathbb{R}^{N}\right),
\end{gathered}
$$

and with the Lax-Milgram theorem we find the respective solutions $h_{k}$.

Using the ellipticity of $\mathcal{A}_{k}$, employing the $\mathcal{A}_{k}$-harmonicity of the $h_{k}$, applying the $\mathcal{A}$-harmonicity of $g$, and exploiting Hölder's inequality, we then have

$$
\begin{aligned}
\lambda \mid & \left.B_{\rho}\left(\xi_{0}\right)\right|^{-1} \int_{B_{\rho}\left(\xi_{0}\right)}\left|X h_{k}-X g\right|^{2} d \xi \\
& \leq\left|B_{\rho}\left(\xi_{0}\right)\right|^{-1} \int_{B_{\rho}\left(\xi_{0}\right)} \mathcal{A}_{k}\left(X h_{k}-X g, X h_{k}-X g\right) d \xi \\
& =\left|B_{\rho}\left(\xi_{0}\right)\right|^{-1} \int_{B_{\rho}\left(\xi_{0}\right)} \mathcal{A}_{k}\left(X h_{k}, X h_{k}-X g\right) d \xi-\left|B_{\rho}\left(\xi_{0}\right)\right|^{-1} \int_{B_{\rho}\left(\xi_{0}\right)} \mathcal{A}_{k}\left(X g, X h_{k}-X g\right) d \xi \\
& =-\left|B_{\rho}\left(\xi_{0}\right)\right|^{-1} \int_{B_{\rho}\left(\xi_{0}\right)} \mathcal{A}_{k}\left(X g, X h_{k}-X g\right) d \xi \\
& =\left|B_{\rho}\left(\xi_{0}\right)\right|^{-1} \int_{B_{\rho}\left(\xi_{0}\right)}\left(\mathcal{A}-\mathcal{A}_{k}\right)\left(X g, X h_{k}-X g\right) d \xi \\
& \leq\left|\mathcal{A}-\mathcal{A}_{k}\right|\left|B_{\rho}\left(\xi_{0}\right)\right|^{-1} \int_{B_{\rho}\left(\xi_{0}\right)}|X g|\left|X h_{k}-X g\right| d \xi \\
& \leq\left|\mathcal{A}-\mathcal{A}_{k}\right|\left(\left|B_{\rho}\left(\xi_{0}\right)\right|^{-1} \int_{B_{\rho}\left(\xi_{0}\right)}|X g|^{2} d \xi\right)^{1 / 2}\left(\left|B_{\rho}\left(\xi_{0}\right)\right|^{-1} \int_{B_{\rho}\left(\xi_{0}\right)}\left|X h_{k}-X g\right|^{2} d \xi\right)^{1 / 2} \\
& \leq\left|\mathcal{A}-\mathcal{A}_{k}\right|\left(\left|B_{\rho}\left(\xi_{0}\right)\right|^{-1} \int_{B_{\rho}\left(\xi_{0}\right)}\left|X h_{k}-X g\right|^{2} d \xi\right)^{1 / 2} \cdot W^{1 / 2}, W e c o n g l y
\end{aligned}
$$

By the convergence of $\mathcal{A}_{k}$ to $\mathcal{A}$ and the fact that $h_{k}-g \in H W_{0}^{1,2}$, we conclude that $h_{k}$ converges strongly to $g$ in $H W_{0}^{1,2}\left(B_{\rho}\left(\xi_{0}\right), \mathbb{R}^{N}\right)$ and, in particular, we have that $\left\|h_{k}-g\right\|_{L^{2}}$ $\rightarrow 0(k \rightarrow \infty)$. So

$$
\left\|h_{k}-g_{k}\right\|_{L^{2}} \leq\left\|g_{k}-g\right\|_{L^{2}}+\left\|h_{k}-g\right\|_{L^{2}} \rightarrow 0 \quad(k \rightarrow \infty),
$$

and this gives the desired contradiction to (3.5). 
$\mathrm{Xu}$ and Zuily [30] considered the following constant coefficients system involving Hörmander's vector fields:

$$
\sum_{i, j=1}^{k} \sum_{\beta=1}^{N} A_{i j}^{\alpha \beta} X_{i} X_{j} u^{\beta}=0, \quad \alpha=1, \ldots, N,
$$

and proved that weak solutions $u \in H W^{1,2}(\Omega)$ implies $u \in C^{\infty}(\Omega)$. Moreover, they established the following inequality for all $\xi_{0} \in \Omega, R \in(0,1)$ s.t. $B\left(\xi_{0}, R\right) \subset \Omega$ and all $k \in \mathbb{N}$ :

$$
R^{k}\|u\|_{H W^{k, 2}\left(B\left(\xi_{0}, R / 2^{k}\right)\right)} \leq C\|u\|_{L^{2}\left(B\left(\xi_{0}, R\right)\right)},
$$

where $C$ is a constant independent of $\xi_{0}, R$ and $u$.

In view of $\left(u-u_{\xi_{0}, R}\right)$ also being a weak solution to the system (3.6), combining (3.7) and (2.6), it is easy to see that

$$
\sup _{B_{R / 2}\left(\xi_{0}\right)}|X u|^{2} \leq C_{0}\left|B_{R}\left(\xi_{0}\right)\right|^{-1} \int_{B_{R}\left(\xi_{0}\right)}|X u|^{2} d \xi,
$$

where $C_{0}$ is a constant independent of $\xi_{0}, R$ and $u$. Without loss of generality we may take $C_{0} \geq 1$.

LEMMA 3.2. (Caccioppoli-type inequality) Assume that $u \in H W^{1, m}\left(\Omega, \mathbb{R}^{N}\right) \cap L^{\infty}\left(\Omega, \mathbb{R}^{N}\right)$ is a weak solution to the system (1.1) with conditions (H1)-(H3) and (N), and $2 a M<\lambda^{\prime}$. Then for every $\xi_{0}=\left(\xi_{0}^{1}, \xi_{0}^{2}\right) \in \Omega \subset \mathbb{R}^{k} \times \mathbb{R}^{n-k}, u_{0} \in \mathbb{R}^{N}, p_{0} \in \mathbb{R}^{k N}$ and any $\rho, R$ satisfying $0<\rho<R<\min \left\{1\right.$, $\left.\operatorname{dist}\left(\xi_{0}, \partial \Omega\right)\right\}$, it holds that

$$
\begin{aligned}
& \int_{B_{\rho}\left(\xi_{0}\right)}\left|X u-p_{0}\right|^{2}+\left|X u-p_{0}\right|^{m} d \xi \\
& \leq C\left[(R-\rho)^{-2} \int_{B_{R}\left(\xi_{0}\right)}\left|u-u_{0}-\left(\xi^{1}-\xi_{0}^{1}\right) p_{0}\right|^{2} d \xi\right. \\
& \quad+(R-\rho)^{-m} \int_{B_{R}\left(\xi_{0}\right)}\left|u-u_{0}-\left(\xi^{1}-\xi_{0}^{1}\right) p_{0}\right|^{m} d \xi \\
& \left.\quad+\left|B_{R}\left(\xi_{0}\right)\right|\left\{\left[K\left(\left|u_{0}\right|+\left|p_{0}\right|\right)\left(1+\left|p_{0}\right|^{2}\right)^{m / 2}\right]^{2 /(1-\gamma)} R^{2 \gamma}+\left(b^{2}+a^{2}\left|p_{0}\right|^{2 m}\right) R^{2}\right\}\right],
\end{aligned}
$$

where $\xi^{1} \in \Omega \subset \mathbb{R}^{k}$ is the component of $\xi=\left(\xi^{1}, \xi^{2}\right) \in \Omega \subset \mathbb{R}^{k} \times \mathbb{R}^{n-k}$.

Proof. Inspired by the method of [13], we let $v=u-u_{0}-\left(\xi^{1}-\xi_{0}^{1}\right) p_{0}$. Take a test function $\varphi=\eta^{2} v$ in (1.10) with $\eta \in C_{0}^{\infty}\left(B_{R}\left(\xi_{0}\right)\right)$ satisfying $0 \leq \eta \leq 1 ; \eta \equiv 1, \forall \xi \in B_{\rho}\left(\xi_{0}\right)$ and $|\nabla \eta|<C(R-\rho)^{-1}$. Then $X v=X u-p_{0}$ from (2.3), and

$$
\begin{aligned}
& \int_{B_{R}\left(\xi_{0}\right)} A_{i}^{\alpha}(\xi, u, X u) \eta^{2}\left(X u-p_{0}\right) d \xi \\
& \quad=-2 \int_{B_{R}\left(\xi_{0}\right)} \eta X \eta A_{i}^{\alpha}(\xi, u, X u) v d \xi+\int_{B_{R}\left(\xi_{0}\right)} B^{\alpha}(\xi, u, X u) \varphi^{\alpha} d \xi .
\end{aligned}
$$


One obtains from the selection of $\varphi$ that

$$
\begin{aligned}
& -\int_{B_{R}\left(\xi_{0}\right)} A_{i}^{\alpha}\left(\xi, u, p_{0}\right) \eta^{2}\left(X u-p_{0}\right) d \xi \\
& \quad=2 \int_{B_{R}\left(\xi_{0}\right)} \eta X \eta A_{i}^{\alpha}\left(\xi, u, p_{0}\right) v d \xi-\int_{B_{R}\left(\xi_{0}\right)} A_{i}^{\alpha}\left(\xi, u, p_{0}\right) X \varphi^{\alpha} d \xi
\end{aligned}
$$

Noting that $A_{i}^{\alpha}\left(\xi_{0}, u_{0}, p_{0}\right)$ is a constant gives

$$
\int_{B_{R}\left(\xi_{0}\right)} A_{i}^{\alpha}\left(\xi_{0}, u_{0}, p_{0}\right) X \varphi^{\alpha} d \xi=0 .
$$

Adding (3.10)-(3.12) and then using (1.4)-(1.6) yields

$$
\begin{aligned}
& \int_{B_{R}\left(\xi_{0}\right)}\left[A_{i}^{\alpha}(\xi, u, X u)-A_{i}^{\alpha}\left(\xi, u, p_{0}\right)\right] \eta^{2}\left(X u-p_{0}\right) d \xi \\
& =-2 \int_{B_{R}\left(\xi_{0}\right)}\left[A_{i}^{\alpha}(\xi, u, X u)-A_{i}^{\alpha}\left(\xi, u, p_{0}\right)\right] \eta v X \eta d \xi \\
& -\int_{B_{R}\left(\xi_{0}\right)} A_{i}^{\alpha}\left(\xi, u, p_{0}\right) X \varphi^{\alpha} d \xi+\int_{B_{R}\left(\xi_{0}\right)} A_{i}^{\alpha}\left(\xi_{0}, u_{0}, p_{0}\right) X \varphi^{\alpha} d \xi \\
& +\int_{B_{R}\left(\xi_{0}\right)} B^{\alpha}(\xi, u, X u) \varphi^{\alpha} d \xi \\
& =2 \int_{B_{R}\left(\xi_{0}\right)}\left[A_{i}^{\alpha}\left(\xi, u, p_{0}\right)-A_{i}^{\alpha}(\xi, u, X u)\right] \eta v X \eta d \xi \\
& +\int_{B_{R}\left(\xi_{0}\right)}\left[A_{i}^{\alpha}\left(\xi, u_{0}+\left(\xi^{1}-\xi_{0}^{1}\right) p_{0}, p_{0}\right)-A_{i}^{\alpha}\left(\xi, u, p_{0}\right)\right] X \varphi^{\alpha} d \xi \\
& +\int_{B_{R}\left(\xi_{0}\right)}\left[A_{i}^{\alpha}\left(\xi_{0}, u_{0}, p_{0}\right)-A_{i}^{\alpha}\left(\xi, u_{0}+\left(\xi^{1}-\xi_{0}^{1}\right) p_{0}, p_{0}\right)\right] X \varphi^{\alpha} d \xi \\
& +\int_{B_{R}\left(\xi_{0}\right)} B^{\alpha}(\xi, u, X u) \varphi^{\alpha} d \xi \\
& \leq 2 C \int_{B_{R}\left(\xi_{0}\right)}\left(1+|X u|^{2}+\left|p_{0}\right|^{2}\right)^{(m-2) / 2}\left|X u-p_{0}\right||\eta\|v\| X \eta| d \xi \\
& +K(\cdot) \int_{B_{R}\left(\xi_{0}\right)}\left|u-u_{0}-\left(\xi^{1}-\xi_{0}^{1}\right) p_{0}\right|^{\gamma}\left(1+\left|p_{0}\right|^{2}\right)^{(m-1) / 2} \eta^{2}|X v| d \xi \\
& +2 K(\cdot) \int_{B_{R}\left(\xi_{0}\right)}\left|u-u_{0}-\left(\xi^{1}-\xi_{0}^{1}\right) p_{0}\right|^{\gamma}\left(1+\left|p_{0}\right|^{2}\right)^{(m-1) / 2}|\eta||v||X \eta| d \xi \\
& +K(\cdot) \int_{B_{R}\left(\xi_{0}\right)}\left[\left(\varrho_{\mathrm{cc}}^{m}\left(\xi_{0}, \xi\right)+\left|\left(\xi^{1}-\xi_{0}^{1}\right) p_{0}\right|^{m}\right)^{\gamma / m}\left(1+\left|p_{0}\right|^{2}\right)^{(m-1) / 2}\right. \\
& \left.\times\left(\eta^{2}|X v|+2|\eta||v||X \eta|\right)\right] d \xi \\
& +\int_{B_{R}\left(\xi_{0}\right)}\left(a|X u|^{m}+b\right)|v| \eta^{2} d \xi \\
& :=\mathrm{I}+\mathrm{II}+\mathrm{III}+\mathrm{IV}+\mathrm{V}
\end{aligned}
$$


where $K(\cdot)=: K\left(\left|u_{0}\right|+\left|\xi^{1}-\xi_{0}^{1}\right|\left|p_{0}\right|\right)$.

Employing (1.8), the left-hand side in (3.13) can be estimated by

$$
\begin{aligned}
& \int_{B_{R}\left(\xi_{0}\right)}\left[A_{i}^{\alpha}(\xi, u, X u)-A_{i}^{\alpha}\left(\xi, u, p_{0}\right)\right]\left(X u-p_{0}\right) \eta^{2} d \xi \\
& \geq \lambda^{\prime}\left[\int_{B_{R}\left(\xi_{0}\right)}\left|X u-p_{0}\right|^{2} \eta^{2} d \xi+\int_{B_{R}\left(\xi_{0}\right)}\left|X u-p_{0}\right|^{m} \eta^{2} d \xi\right] .
\end{aligned}
$$

In what follows, we will estimate the five terms of the right-hand side in (3.13), respectively.

For a small positive $\varepsilon$ to be fixed later, using Young's inequality and $|X \eta| \leq C(R-\rho)^{-1}$, we have

$$
\begin{aligned}
I \leq & 2 C \int_{B_{R}\left(\xi_{0}\right)}\left(1+3\left|p_{0}\right|^{2}+2\left|X u-p_{0}\right|^{2}\right)^{(m-2) / 2}\left|X u-p_{0}\right||\eta||v \| X \eta| d \xi \\
\leq & 2 C \max \left\{1,2^{(m-4) / 2}\right\}\left[\int_{B_{R}\left(\xi_{0}\right)}\left(1+3\left|p_{0}\right|^{2}\right)^{(m-2) / 2}|\eta||X \eta|\left|X u-p_{0}\right||v| d \xi\right. \\
& \left.+2^{(m-2) / 2} \int_{B_{R}\left(\xi_{0}\right)}|\eta||X \eta|\left|X u-p_{0}\right|^{m-1}|v| d \xi\right] \\
\leq & C \varepsilon \int_{B_{R}\left(\xi_{0}\right)}|\eta|^{2}\left|X u-p_{0}\right|^{2} d \xi+C \varepsilon^{-1}(R-\rho)^{-2} \int_{B_{R}\left(\xi_{0}\right)}|v|^{2} d \xi \\
& +C \varepsilon \int_{B_{R}\left(\xi_{0}\right)}\left|X u-p_{0}\right|^{m}|\eta|^{m /(m-1)} d \xi+C \varepsilon^{1-m}(R-\rho)^{-m} \int_{B_{R}\left(\xi_{0}\right)}|v|^{m} d \xi .
\end{aligned}
$$

Noting the fact that $(R / R-\rho)^{2 \gamma} \geq 1$ yields

$$
\begin{aligned}
\mathrm{II} \leq & K\left(\left|u_{0}\right|+R\left|p_{0}\right|\right) \int_{B_{R}\left(\xi_{0}\right)}|v|^{\gamma}\left(1+\left|p_{0}\right|^{2}\right)^{(m-1) / 2} \eta^{2}\left|X u-p_{0}\right| d \xi \\
\leq & \varepsilon \int_{B_{R}\left(\xi_{0}\right)} \eta^{4}\left|X u-p_{0}\right|^{2} d \xi+\varepsilon^{-1}\left(1+\left|p_{0}\right|^{2}\right)^{m} K^{2}\left(\left|u_{0}\right|+\left|p_{0}\right|\right) \\
& \times \int_{B_{R}\left(\xi_{0}\right)}|v|^{2 \gamma}\left(\frac{R}{R-\rho}\right)^{2 \gamma} d \xi \\
\leq & \varepsilon \int_{B_{R}\left(\xi_{0}\right)} \eta^{2}\left|X u-p_{0}\right|^{2} d \xi+\varepsilon^{-1} \int_{B_{R}\left(\xi_{0}\right)}|v|^{2}(R-\rho)^{-2} d \xi \\
& +\varepsilon^{-1}\left[K\left(\left|u_{0}\right|+\left|p_{0}\right|\right)\left(1+\left|p_{0}\right|^{2}\right)^{m / 2}\right]^{2 /(1-\gamma)} R^{2 \gamma /(1-\gamma)}\left|B_{R}\left(\xi_{0}\right)\right| .
\end{aligned}
$$

Similarly, it follows that

$$
\begin{aligned}
\mathrm{III} \leq & 2 K\left(\left|u_{0}\right|+\left|p_{0}\right|\right)\left(1+\left|p_{0}\right|^{2}\right)^{(m-1) / 2} \int_{B_{R}\left(\xi_{0}\right)}|v|^{\gamma}|\eta||X \eta||v| d \xi \\
\leq & 4 \int_{B_{R}\left(\xi_{0}\right)}|v|^{2}|X \eta|^{2} d \xi+K^{2}\left(\left|u_{0}\right|+\left|p_{0}\right|\right)\left(1+\left|p_{0}\right|^{2}\right)^{m} \\
& \times \int_{B_{R}\left(\xi_{0}\right)}|v|^{2 \gamma}\left(\frac{R}{R-\rho}\right)^{2 \gamma}|\eta|^{2} d \xi
\end{aligned}
$$




$$
\begin{aligned}
\leq & C \int_{B_{R}\left(\xi_{0}\right)}|v|^{2}(R-\rho)^{-2} d \xi \\
& +\left[K\left(\left|u_{0}\right|+\left|p_{0}\right|\right)\left(1+\left|p_{0}\right|^{2}\right)^{m / 2}\right]^{2 /(1-\gamma)} R^{2 \gamma /(1-\gamma)}\left|B_{R}\left(\xi_{0}\right)\right|
\end{aligned}
$$

and

$$
\begin{aligned}
\mathrm{IV} \leq & K\left(\left|u_{0}\right|+\left|p_{0}\right|\right) \\
& \times \int_{B_{R}\left(\xi_{0}\right)} R^{\gamma}\left(1+\left|p_{0}\right|^{m}\right)^{\gamma / m}\left(1+\left|p_{0}\right|^{2}\right)^{(m-1) / 2}\left(\eta^{2}\left|X u-p_{0}\right|+2|\eta||v||X \eta|\right) d \xi \\
\leq & K\left(\left|u_{0}\right|+\left|p_{0}\right|\right) \int_{B_{R}\left(\xi_{0}\right)} R^{\gamma}\left(1+\left|p_{0}\right|^{2}\right)^{m / 2+\gamma}\left(\eta^{2}\left|X u-p_{0}\right|+2|\eta||v||X \eta|\right) d \xi \\
\leq & \varepsilon \int_{B_{R}\left(\xi_{0}\right)} \eta^{4}\left|X u-p_{0}\right|^{2} d \xi+\int_{B_{R}\left(\xi_{0}\right)}|v|^{2}|X \eta|^{2} d \xi \\
& +\left(\varepsilon^{-1}+4\right) K^{2}\left(\left|u_{0}\right|+\left|p_{0}\right|\right)\left(1+\left|p_{0}\right|^{2}\right)^{m+2 \gamma} R^{2 \gamma}\left|B_{R}\left(\xi_{0}\right)\right| \\
\leq & \varepsilon \int_{B_{R}\left(\xi_{0}\right)}\left|X u-p_{0}\right|^{2} \eta^{2} d \xi+C(R-\rho)^{-2} \int_{B_{R}\left(\xi_{0}\right)}|v|^{2} d \xi \\
& +\left(\varepsilon^{-1}+4\right) K^{2}\left(\left|u_{0}\right|+\left|p_{0}\right|\right)\left(1+\left|p_{0}\right|^{2}\right)^{m+2 \gamma} R^{2 \gamma}\left|B_{R}\left(\xi_{0}\right)\right| .
\end{aligned}
$$

For a small positive $\mu$ to be fixed later, we have

$$
\begin{aligned}
\mathrm{V} \leq & \int_{B_{R}\left(\xi_{0}\right)}\left(a|X u|^{m}+b\right)|v| \eta^{2} d \xi \\
\leq & \int_{B_{R}\left(\xi_{0}\right)} a|X u|^{m}|v| \eta^{2} d \xi+\int_{B_{R}\left(\xi_{0}\right)} b|v| \eta^{2}\left(\frac{R}{R-\rho}\right) d \xi \\
\leq & \int_{B_{R}\left(\xi_{0}\right)} a\left[(1+\mu)\left|X u-p_{0}\right|^{m}+\left(1+\mu^{-1}\right)\left|p_{0}\right|^{m}\right]|v| \eta^{2} d \xi \\
& +\frac{1}{2} \varepsilon b^{2} R^{2}\left|B_{R}\left(\xi_{0}\right)\right|+\frac{1}{2} \varepsilon^{-1} \int_{B_{R}\left(\xi_{0}\right)}|v|^{2}(R-\rho)^{-2} d \xi \\
\leq & a(1+\mu)\left(2 M+R\left|p_{0}\right|\right) \int_{B_{R}\left(\xi_{0}\right)}\left|X u-p_{0}\right|^{m} \eta^{2} d \xi \\
& +a\left(1+\mu{ }^{-1}\right)\left|p_{0}\right|^{m} \int_{B_{R}\left(\xi_{0}\right)}|v| \eta^{2}\left(\frac{R}{R-\rho}\right) d \xi \\
& +\frac{1}{2} \varepsilon b^{2} R^{2}\left|B_{R}\left(\xi_{0}\right)\right|+\frac{1}{2} \varepsilon^{-1} \int_{B_{R}\left(\xi_{0}\right)}|v|^{2}(R-\rho)^{-2} d \xi \\
\leq & a(1+\mu)\left(2 M+R\left|p_{0}\right|\right) \int_{B_{R}\left(\xi_{0}\right)}\left|X u-p_{0}\right|^{m} \eta^{2} d \xi \\
& +\varepsilon^{-1}(R-\rho)^{-2} \int_{B_{R}\left(\xi_{0}\right)}|v|^{2} d \xi \\
& +\frac{1}{2} \varepsilon\left[b^{2}+a^{2}\left(1+\mu^{-1}\right)^{2}\left|p_{0}\right|^{2 m}\right] R^{2}\left|B_{R}\left(\xi_{0}\right)\right|,
\end{aligned}
$$


where we have used the fact that

$$
|v|=\left|u-u_{0}-\left(\xi^{1}-\xi_{0}^{1}\right) p_{0}\right| \leq 2 \sup _{B_{R}\left(\xi_{0}\right)}|u|+R\left|p_{0}\right| \leq 2 M+R\left|p_{0}\right| .
$$

Paying attention to the facts that

$$
K^{2} \leq K^{2 /(1-\gamma)}(K \geq 1), \quad R^{2 \gamma /(1-\gamma)} \leq R^{2 \gamma}
$$

and

$$
\left(1+\left|p_{0}\right|^{2}\right)^{m+2 \gamma} \leq\left(1+\left|p_{0}\right|^{2}\right)^{m /(1-\gamma)},
$$

and substituting (3.14)-(3.19) into (3.13), it is not difficult to obtain

$$
\begin{aligned}
\Lambda & {\left[\int_{B_{\rho}\left(\xi_{0}\right)}\left|X u-p_{0}\right|^{2} d \xi+\int_{B_{\rho}\left(\xi_{0}\right)}\left|X u-p_{0}\right|^{m} d \xi\right] } \\
\leq & \widehat{\Lambda}(R-\rho)^{-2} \int_{B_{R}\left(\xi_{0}\right)}|v|^{2} d \xi+C \varepsilon^{1-m}(R-\rho)^{-m} \int_{B_{R}\left(\xi_{0}\right)}|v|^{m} d \xi \\
& +\left(2 \varepsilon^{-1}+5\right)\left[K\left(\left|u_{0}\right|+\left|p_{0}\right|\right)\left(1+\left|p_{0}\right|^{2}\right)^{m / 2}\right]^{2 /(1-\gamma)} R^{2 \gamma}\left|B_{R}\left(\xi_{0}\right)\right| \\
& +\frac{1}{2} \varepsilon\left[b^{2}+a^{2}\left(1+\mu^{-1}\right)^{2}\left|p_{0}\right|^{2 m}\right] R^{2}\left|B_{R}\left(\xi_{0}\right)\right|,
\end{aligned}
$$

where $\Lambda=\lambda^{\prime}-2(1+C) \varepsilon-a(1+\mu)\left(2 M+R\left|p_{0}\right|\right), \widehat{\Lambda}=\left(C \varepsilon^{-1}+2 \varepsilon^{-1}+1+C\right)$. The conclusion follows by choosing suitable $\varepsilon$ and $\mu$, such that $\Lambda>0$. We refer to [12] for a similar and detailed argument.

Remark 3.3. The function $v$ chosen in the proof is essentially different from that in [6].

\section{Proof of Theorem 1.1}

We will complete the proof of Theorem 1.1 via four steps.

First step. We claim that if $\rho \leq 1$ and $\varphi \in C_{0}^{\infty}\left(B_{\rho}\left(\xi_{0}\right), \mathbb{R}^{N}\right)$ with $\sup _{B_{\rho}\left(\xi_{0}\right)}|X \varphi| \leq 1$, then there exists a constant $C_{1}=C_{1}(m, Q, a, b)>1$ such that

$$
\begin{aligned}
& \left|B_{\rho}\left(\xi_{0}\right)\right|^{-1} \int_{B_{\rho}\left(\xi_{0}\right)} A_{i, p_{\beta}^{j}}^{\alpha}\left(\xi_{0}, u_{0}, p_{0}\right)\left(X u-p_{0}\right) X \varphi^{\alpha} d \xi \\
& \quad \leq C_{1} \sup _{B_{\rho}\left(\xi_{0}\right)}|X \varphi|\left[\omega^{1 / m}\left(\left|p_{0}\right|, \Phi^{1 / m}\left(\xi_{0}, \rho, p_{0}\right)\right) \Phi^{1 / 2}\left(\xi_{0}, \rho, p_{0}\right)\right. \\
& \left.\quad+\Phi\left(\xi_{0}, \rho, p_{0}\right)+\rho^{\gamma} H\left(\left|u_{0}\right|+\left|p_{0}\right|\right)\right],
\end{aligned}
$$

where we denote $H(\tau)=\left[\max \{K(\tau), a, b\}\left(1+\tau^{2}\right)^{m / 2}\right]^{m /(1-\gamma)}$.

Proof. Noting the fact that

$$
\begin{gathered}
\int_{B_{\rho}\left(\xi_{0}\right)}\left[\int_{0}^{1} A_{i, p_{\beta}^{j}}^{\alpha}\left(\xi_{0}, u_{0}, \theta X u+(1-\theta) p_{0}\right)\left(X u-p_{0}\right) d \theta\right] X \varphi^{\alpha} d \xi \\
=\int_{B_{\rho}\left(\xi_{0}\right)}\left[\int_{0}^{1} \frac{d}{d \theta} A_{i}^{\alpha}\left(\xi_{0}, u_{0}, \theta X u+(1-\theta) p_{0}\right) d \theta\right] X \varphi^{\alpha} d \xi
\end{gathered}
$$




$$
\begin{aligned}
= & \int_{B_{\rho}\left(\xi_{0}\right)}\left[A_{i}^{\alpha}\left(\xi_{0}, u_{0}, X u\right)-A_{i}^{\alpha}\left(\xi_{0}, u_{0}, p_{0}\right)\right] X \varphi^{\alpha} d \xi \\
= & \int_{B_{\rho}\left(\xi_{0}\right)}\left[A_{i}^{\alpha}\left(\xi_{0}, u_{0}, X u\right)-A_{i}^{\alpha}(\xi, u, X u)\right] X \varphi^{\alpha} d \xi \\
& +\int_{B_{\rho}\left(\xi_{0}\right)} B^{\alpha}(\xi, u, X u) \varphi^{\alpha} d \xi,
\end{aligned}
$$

where we have used (3.12) over the ball $B_{\rho}\left(\xi_{0}\right)$ and (1.10), we obtain

$$
\begin{aligned}
& \int_{B_{\rho}\left(\xi_{0}\right)} A_{i, p_{\beta}^{j}}^{\alpha}\left(\xi_{0}, u_{0}, p_{0}\right)\left(X u-p_{0}\right) X \varphi^{\alpha} d \xi \\
& =\int_{B_{\rho}\left(\xi_{0}\right)}\left\{\int_{0}^{1}\left[A_{i, p_{\beta}^{j}}^{\alpha}\left(\xi_{0}, u_{0}, p_{0}\right)-A_{i, p_{\beta}^{j}}^{\alpha}\left(\xi_{0}, u_{0}, \theta X u+(1-\theta) p_{0}\right)\right]\right. \\
& \left.\quad \times\left(X u-p_{0}\right) d \theta\right\} X \varphi^{\alpha} d \xi \\
& \quad+\int_{B_{\rho}\left(\xi_{0}\right)}\left[A_{i}^{\alpha}\left(\xi_{0}, u_{0}, X u\right)-A_{i}^{\alpha}(\xi, u, X u)\right] X \varphi^{\alpha} d \xi \\
& \quad+\int_{B_{\rho}\left(\xi_{0}\right)} B^{\alpha}(\xi, u, X u) \varphi^{\alpha} d \xi
\end{aligned}
$$

Using (1.1) and (1.7) gives

$$
\begin{aligned}
&\left|A_{i, p_{\beta}^{j}}^{\alpha}\left(\xi_{0}, u_{0}, p_{0}\right)-A_{i, p_{\beta}^{j}}^{\alpha}\left(\xi_{0}, u_{0}, \theta X u+(1-\theta) p_{0}\right)\right|^{1 / m+(1-1 / m)} \\
& \leq {\left[C\left(1+\left|p_{0}\right|^{2}+\left|\theta\left(X u-p_{0}\right)+p_{0}\right|^{2}\right)^{(m-2) / 2} \omega\left(\left|p_{0}\right|,\left|\theta\left(X u-p_{0}\right)\right|\right)\right]^{1 / m} } \\
& \times\left[C\left(1+\left|p_{0}\right|^{2}\right)^{(m-2) / 2}+C\left(1+\left|\theta\left(X u-p_{0}\right)+p_{0}\right|^{2}\right)^{(m-2) / 2}\right]^{1-1 / m} \\
& \leq {\left[C\left(1+3\left|p_{0}\right|^{2}+2\left|X u-p_{0}\right|^{2}\right)^{(m-2) / 2} \omega\left(\left|p_{0}\right|,\left|X u-p_{0}\right|\right)\right]^{1 / m} } \\
& \times\left[2 C\left(1+2\left|p_{0}\right|^{2}+2\left|X u-p_{0}\right|^{2}\right)^{(m-2) / 2}\right]^{1-1 / m} \\
& \leq 4 C\left(1+\left|p_{0}\right|^{2}\right)^{(m-2) / 2}\left(1+\left|X u-p_{0}\right|^{2}\right)^{(m-2) / 2} \omega^{1 / m}\left(\left|p_{0}\right|,\left|X u-p_{0}\right|\right)
\end{aligned}
$$

Substituting (4.4) into (4.3) yields

$$
\begin{aligned}
& \left|\int_{B_{\rho}\left(\xi_{0}\right)} A_{i, p_{\beta}^{j}}^{\alpha}\left(\xi_{0}, u_{0}, p_{0}\right)\left(X u-p_{0}\right) X \varphi^{\alpha} d \xi\right| \\
& \quad \leq C \int_{B_{\rho}\left(\xi_{0}\right)} \omega^{1 / m}\left(\left|p_{0}\right|,\left|X u-p_{0}\right|\right)\left(1+\left|X u-p_{0}\right|^{2}\right)^{(m-2) / 2}\left|X u-p_{0}\right| \sup _{B_{\rho}\left(\xi_{0}\right)}|X \varphi| d \xi \\
& \quad+\int_{B_{\rho}\left(\xi_{0}\right)}\left|A_{i}^{\alpha}\left(\xi_{0}, u_{0}, X u\right)-A_{i}^{\alpha}\left(\xi, u_{0}+p_{0}\left(\xi^{1}-\xi_{0}^{1}\right), X u\right)\right| \sup _{B_{\rho}\left(\xi_{0}\right)}|X \varphi| d \xi
\end{aligned}
$$




$$
\begin{aligned}
& \quad+\int_{B_{\rho}\left(\xi_{0}\right)}\left|A_{i}^{\alpha}\left(\xi, u_{0}+p_{0}\left(\xi^{1}-\xi_{0}^{1}\right), X u\right)-A_{i}^{\alpha}(\xi, u, X u)\right| \sup _{B_{\rho}\left(\xi_{0}\right)}|X \varphi| d \xi \\
& \quad+\int_{B_{\rho}\left(\xi_{0}\right)}\left(a|X u|^{m}+b\right)|\varphi| d \xi \\
& :=\mathrm{I}^{\prime}+\mathrm{II}^{\prime}+\mathrm{III}^{\prime}+\mathrm{IV}^{\prime} .
\end{aligned}
$$

By Hölder's inequality, Jensen's inequality and $m /(m-1) \leq 2$, it follows that

$$
\begin{aligned}
& \mathrm{I}^{\prime} \leq C\left|B_{\rho}\left(\xi_{0}\right)\right|\left[\left|B_{\rho}\left(\xi_{0}\right)\right|^{-1} \int_{B_{\rho}\left(\xi_{0}\right)} \omega\left(\left|p_{0}\right|,\left|X u-p_{0}\right|\right) d \xi\right]^{1 / m} \\
& \times\left\{\left|B_{\rho}\left(\xi_{0}\right)\right|^{-1} \int_{B_{\rho}\left(\xi_{0}\right)}\left[\left(1+\left|X u-p_{0}\right|^{2}\right)^{(m-2) / 2}\left|X u-p_{0}\right|\right]^{m /(m-1)} d \xi\right\}^{(m-1) / m} \\
& \leq C\left|B_{\rho}\left(\xi_{0}\right)\right| \omega^{1 / m}\left(\left|p_{0}\right|,\left|B_{\rho}\left(\xi_{0}\right)\right|^{-1} \int_{B_{\rho}\left(\xi_{0}\right)}\left|X u-p_{0}\right| d \xi\right) \\
& \times\left[\left|B_{\rho}\left(\xi_{0}\right)\right|^{-1} \int_{B_{\rho}\left(\xi_{0}\right)}\left|X u-p_{0}\right|^{m /(m-1)} d \xi\right. \\
& \left.+\left|B_{\rho}\left(\xi_{0}\right)\right|^{-1} \int_{B_{\rho}\left(\xi_{0}\right)}\left|X u-p_{0}\right|^{m} d \xi\right]^{(m-1) / m} \\
& \leq C\left|B_{\rho}\left(\xi_{0}\right)\right| \omega^{1 / m}\left(\left|p_{0}\right|,\left(\left|B_{\rho}\left(\xi_{0}\right)\right|^{-1} \int_{B_{\rho}\left(\xi_{0}\right)}\left|X u-p_{0}\right|^{m} d \xi\right)^{1 / m}\right) \\
& \times\left[\left(\left|B_{\rho}\left(\xi_{0}\right)\right|^{-1} \int_{B_{\rho}\left(\xi_{0}\right)}\left|X u-p_{0}\right|^{m /(m-1)} d \xi\right)^{(m-1) / m}\right. \\
& \left.+\left(\left|B_{\rho}\left(\xi_{0}\right)\right|^{-1} \int_{B_{\rho}\left(\xi_{0}\right)}\left|X u-p_{0}\right|^{m} d \xi\right)^{(m-1) / m}\right] \\
& \leq C\left|B_{\rho}\left(\xi_{0}\right)\right| \omega^{1 / m}\left(\left|p_{0}\right|,\left(\left|B_{\rho}\left(\xi_{0}\right)\right|^{-1} \int_{B_{\rho}\left(\xi_{0}\right)}\left|X u-p_{0}\right|^{m} d \xi\right)^{1 / m}\right) \\
& \times\left[\left(\left|B_{\rho}\left(\xi_{0}\right)\right|^{-1} \int_{B_{\rho}\left(\xi_{0}\right)}\left|X u-p_{0}\right|^{2} d \xi\right)^{1 / 2}\right. \\
& \left.+\left(\left|B_{\rho}\left(\xi_{0}\right)\right|^{-1} \int_{B_{\rho}\left(\xi_{0}\right)}\left|X u-p_{0}\right|^{m} d \xi\right)^{(m-1) / m}\right] \\
& \leq C\left|B_{\rho}\left(\xi_{0}\right)\right| \omega^{1 / m}\left(\left|p_{0}\right|,\left(\left|B_{\rho}\left(\xi_{0}\right)\right|^{-1} \int_{B_{\rho}\left(\xi_{0}\right)}\left|X u-p_{0}\right|^{m} d \xi\right)^{1 / m}\right) \\
& \times\left[\left|B_{\rho}\left(\xi_{0}\right)\right|^{-1} \int_{B_{\rho}\left(\xi_{0}\right)}\left|X u-p_{0}\right|^{2}+\left|X u-p_{0}\right|^{m} d \xi\right]^{1 / 2} \\
& \leq C\left|B_{\rho}\left(\xi_{0}\right)\right| \omega^{1 / m}\left(\left|p_{0}\right|, \Phi^{1 / m}\left(\xi_{0}, \rho, p_{0}\right)\right) \Phi^{1 / 2}\left(\xi_{0}, \rho, p_{0}\right) \text {. }
\end{aligned}
$$


We use (1.4), Young's inequality, $K \leq K^{2 /(1-\gamma)}, \rho^{\gamma} \leq 1$ and $2 \gamma \leq m / 2+\gamma \leq m /(1-\gamma)$ to obtain

$$
\begin{aligned}
\mathrm{II}^{\prime} \leq & \int_{B_{\rho}\left(\xi_{0}\right)} K\left(\left|u_{0}\right|\right) \rho^{\gamma}\left(1+\left|p_{0}\right|^{m}\right)^{\gamma / m}\left(1+|X u|^{2}\right)^{(m-1) / 2} d \xi \\
\leq & 2^{m / 2-1} C \int_{B_{\rho}\left(\xi_{0}\right)} K\left(\left|u_{0}\right|+\left|p_{0}\right|\right) \rho^{\gamma}\left(1+\left|p_{0}\right|^{m}\right)^{\gamma / m} \\
& \times\left[\left(1+\left|p_{0}\right|^{2}\right)^{m / 2}+\left(\left|X u-p_{0}\right|^{2}\right)^{m / 2}\right] d \xi \\
\leq & C K\left(\left|u_{0}\right|+\left|p_{0}\right|\right) \rho^{\gamma}\left(1+\left|p_{0}\right|\right)^{m / 2+\gamma}\left|B_{\rho}\left(\xi_{0}\right)\right|+C \int_{B_{\rho}\left(\xi_{0}\right)}\left|X u-p_{0}\right|^{m} d \xi \\
\leq & C\left|B_{\rho}\left(\xi_{0}\right)\right|\left[\Phi\left(\xi_{0}, \rho, p_{0}\right)+K\left(\left|u_{0}\right|+\left|p_{0}\right|\right) \rho^{\gamma}\left(1+\left|p_{0}\right|^{2}\right)^{m / 2+\gamma}\right] \\
\leq & C\left|B_{\rho}\left(\xi_{0}\right)\right|\left\{\Phi\left(\xi_{0}, \rho, p_{0}\right)+\left[K\left(\left|u_{0}\right|+\left|p_{0}\right|\right)\left(1+\left|p_{0}\right|^{2}\right)^{m / 2}\right]^{2 /(1-\gamma)} \rho^{\gamma}\right\} .
\end{aligned}
$$

It follows from Young's inequality, (2.6) and $\rho^{2 \gamma /(1-\gamma)}, \rho^{2 \gamma /(2-\gamma)} \leq \rho^{\gamma}$ that

$$
\begin{aligned}
\mathrm{III}^{\prime} \leq & \int_{B_{\rho}\left(\xi_{0}\right)} K\left(\left|u_{0}\right|+\left|\rho p_{0}\right|\right)|v|^{\gamma}\left(1+|X u|^{2}\right)^{(m-1) / 2} d \xi \\
\leq & C \int_{B_{\rho}\left(\xi_{0}\right)} K\left(\left|u_{0}\right|+\left|p_{0}\right|\right)\left(1+\left|p_{0}\right|^{2}\right)^{m / 2}|v|^{\gamma} d \xi \\
& +C \int_{B_{\rho}\left(\xi_{0}\right)} K\left(\left|u_{0}\right|+\left|p_{0}\right|\right)\left|X u-p_{0}\right|^{m-1}|v|^{\gamma} d \xi \\
\leq & C\left\{\int_{B_{\rho}\left(\xi_{0}\right)} \rho^{-2}|v|^{2} d \xi+\left|B_{\rho}\left(\xi_{0}\right)\right|\left[K\left(\left|u_{0}\right|+\left|p_{0}\right|\right)\left(1+\left|p_{0}\right|^{2}\right)^{m / 2} \rho^{\gamma}\right]^{2 /(2-\gamma)}\right\} \\
& +C\left[\int_{B_{\rho}\left(\xi_{0}\right)}\left|X u-p_{0}\right|^{m} d \xi+\int_{B_{\rho}\left(\xi_{0}\right)} K^{m}\left(\left|u_{0}\right|+\left|p_{0}\right|\right) \rho^{m \gamma}\left(|v|^{m \gamma} \rho^{-m \gamma}\right) d \xi\right] \\
\leq & C\left\{\int_{B_{\rho}\left(\xi_{0}\right)} \rho^{-2}|v|^{2} d \xi+\left|B_{\rho}\left(\xi_{0}\right)\right|\left[K\left(\left|u_{0}\right|+\left|p_{0}\right|\right)\left(1+\left|p_{0}\right|^{2}\right)^{m / 2}\right]^{m /(1-\gamma)} \rho^{\gamma}\right. \\
& \left.+\int_{B_{\rho}\left(\xi_{0}\right)}\left|X u-p_{0}\right|^{m} d \xi+\int_{B_{\rho}\left(\xi_{0}\right)} \rho^{-m}|v|^{m} d \xi\right\} \\
\leq & C\left\{\left|B_{\rho}\left(\xi_{0}\right)\right|\left[K\left(\left|u_{0}\right|+\left|p_{0}\right|\right)\left(1+\left|p_{0}\right|^{2}\right)^{m / 2}\right]^{m /(1-\gamma)} \rho^{\gamma}\right. \\
& \left.+C_{P} \int_{B_{\rho}\left(\xi_{0}\right)}\left|X u-p_{0}\right|^{2} d \xi+\left(1+C_{P}\right) \int_{B_{\rho}\left(\xi_{0}\right)}\left|X u-p_{0}\right|^{m} d \xi\right\} \\
\leq & C\left|B_{\rho}\left(\xi_{0}\right)\right|\left\{\Phi\left(\xi_{0}, \rho, p_{0}\right)+\left[K\left(\left|u_{0}\right|+\left|p_{0}\right|\right)\left(1+\left|p_{0}\right|^{2}\right)^{m / 2}\right]^{m /(1-\gamma)} \rho^{\gamma}\right\} .
\end{aligned}
$$

Noting the fact that $\sup _{B_{\rho}\left(\xi_{0}\right)}|\varphi| \leq \rho \leq 1$, we have

$$
\begin{aligned}
\mathrm{IV}^{\prime} & \leq 2^{m-1} C \rho\left[\int_{B_{\rho}\left(\xi_{0}\right)} a\left|X u-p_{0}\right|^{m} d \xi+\left(a\left|p_{0}\right|^{m}+b\right)\left|B_{\rho}\left(\xi_{0}\right)\right|\right] \\
& \leq C \rho^{\gamma}\left|B_{\rho}\left(\xi_{0}\right)\right|\left[a \Phi\left(\xi_{0}, \rho, p_{0}\right)+\left(a\left|p_{0}\right|^{m}+b\right)\right] .
\end{aligned}
$$

Now, we conclude (4.1) by combining estimates (4.6)-(4.9) with (4.5). 
Second step. We claim that for a fixed $\gamma \in(0,1)$, there exist positive constants $C_{2}, C_{3}$ and $\delta$ satisfying the conditions in Lemma 3.1 and $\theta \in(0,1)$ such that

$$
\begin{gathered}
\omega\left(\left|u_{\xi_{0}, \rho}\right|+\left|(X u)_{\xi_{0}, \rho}\right|, \Phi^{1 / m}\left(\xi_{0}, \rho,(X u)_{\xi_{0}, \rho}\right)\right) \leq \frac{\delta^{m}}{4^{m}}, \\
\Phi\left(\xi_{0}, \rho,(X u)_{\xi_{0}, \rho}\right) \leq \min \left\{\frac{\delta^{2}}{16}, \frac{1}{4 C_{2}^{2}}, \frac{M_{1}^{2}\left(1-\theta^{\gamma}\right)^{2} \theta^{Q}}{4\left(1+\sqrt{C_{P}}\right)^{2}}\right\},
\end{gathered}
$$

where $M_{1}$ is a positive constant and $C_{2}=(2 \theta)^{-Q / 2} \sqrt{C_{P}}+C_{1} \sqrt{C_{0}}$ and

$$
2 C_{2} \rho^{\gamma} H\left(1+\left|u_{\xi_{0}, \rho}\right|+\left|(X u)_{\xi_{0}, \rho}\right|\right) \leq \delta / 2 .
$$

The three inequalities above imply

$$
\Phi\left(\xi_{0}, \theta \rho,(X u)_{\xi_{0}, \theta \rho}\right) \leq \theta^{2 \gamma}\left[\frac{1}{2} \Phi\left(\xi_{0}, \rho,(X u)_{\xi_{0}, \rho}\right)+C_{3} \rho^{2 \gamma} H_{0}^{2}\right],
$$

where $H_{0}=H\left(1+M_{1}\right)$.

Proof. Paying attention to the properties of $\omega(s, t)$ in (1.7), we choose a suitable $t_{0}>0$ satisfying

$$
t_{0} \leq \min \left\{\frac{\delta^{2}}{16}, \frac{1}{4 C_{2}^{2}}, \frac{M_{1}^{2}\left(1-\theta^{\gamma}\right)^{2} \theta^{Q}}{4\left(1+\sqrt{C_{P}}\right)^{2}}\right\}
$$

such that

$$
\omega\left(M_{1}, t_{0}\right) \leq \frac{\delta^{m}}{4^{m}}
$$

Moreover, let us take $\rho_{0}>0$ such that $\rho_{0}^{2 \gamma}<t_{0} /\left(4 C_{3}+C_{2}^{2}\right) H_{0}^{2}$, which ensures

$$
2 C_{2} \rho_{0}^{\gamma} H_{0} \leq \delta / 2 \text { and } 2 C_{3} \rho_{0}^{2 \gamma} H_{0}^{2} \leq t_{0} / 2 .
$$

Next, choosing $\rho \in\left(0, \rho_{0}\right]$ satisfying

$$
\left|u \xi_{0}, \rho\right|+\left|(X u)_{\xi_{0}, \rho}\right|<M_{1} / 2, \quad \Phi\left(\xi_{0}, \rho,(X u)_{\xi_{0}, \rho}\right)<t_{0} / 2,
$$

it follows that (4.10)-(4.12) are valid.

In the sequel, let $w=\left[u-u \xi_{0}, \rho-p_{0}\left(\xi^{1}-\xi_{0}^{1}\right)\right] \sigma$, where

$$
\sigma=C_{1}^{-1}\left[\Phi\left(\xi_{0}, \rho, p_{0}\right)+4 \delta^{-2} \rho^{2 \gamma} H^{2}\left(\left|u_{0}\right|+\left|p_{0}\right|\right)\right]^{-1 / 2}
$$

and $p_{0} \in \mathbb{R}^{k N}$ is fixed, then $X w=\left(X u-p_{0}\right) \sigma$. From (4.1), we have for any $\varphi \in$ $C_{0}^{\infty}\left(B_{\rho}\left(\xi_{0}\right), \mathbb{R}^{N}\right)$ that

$$
\begin{aligned}
& \int_{B_{\rho}\left(\xi_{0}\right)}\left[A_{i, p_{j}^{\beta}}^{\alpha}\left(\xi_{0}, u_{0}, p_{0}\right) X w\right] X \varphi^{\alpha} d \xi \\
& \leq C_{1} \sigma\left|B_{\rho}\left(\xi_{0}\right)\right| \sup _{B_{\rho}\left(\xi_{0}\right)}|X \varphi|\left[\omega^{1 / m}\left(\left|p_{0}\right|, \Phi^{1 / m}\left(\xi_{0}, \rho, p_{0}\right)\right) \Phi^{1 / 2}\left(\xi_{0}, \rho, p_{0}\right)\right. \\
& \left.\quad+\Phi\left(\xi_{0}, \rho, p_{0}\right)+\rho^{\gamma} H\left(\left|u_{0}\right|+\left|p_{0}\right|\right)\right] \\
& \leq\left|B_{\rho}\left(\xi_{0}\right)\right|\left\{\Phi ^ { - 1 / 2 } ( \xi _ { 0 } , \rho , p _ { 0 } ) \left[\omega^{1 / m}\left(\left|p_{0}\right|, \Phi^{1 / m}\left(\xi_{0}, \rho, p_{0}\right)\right) \Phi^{1 / 2}\left(\xi_{0}, \rho, p_{0}\right)\right.\right. \\
& \left.\left.\quad+\Phi\left(\xi_{0}, \rho, p_{0}\right)\right]+\left[4 \delta^{-2} \rho^{2 \gamma} H^{2}\left(\left|u_{0}\right|+\left|p_{0}\right|\right)\right]^{-\frac{1}{2}} \rho^{\gamma} H\left(\left|u_{0}\right|+\left|p_{0}\right|\right)\right\} \sup _{B_{\rho}\left(\xi_{0}\right)}|X \varphi| \\
& \leq\left|B_{\rho}\left(\xi_{0}\right)\right|\left[\omega^{1 / m}\left(\left|p_{0}\right|, \Phi^{1 / m}\left(\xi_{0}, \rho, p_{0}\right)\right)+\Phi^{1 / 2}\left(\xi_{0}, \rho, p_{0}\right)+\frac{\delta}{2}\right] \sup _{B_{\rho}\left(\xi_{0}\right)}|X \varphi|
\end{aligned}
$$


and

$$
\begin{aligned}
& \left|B_{\rho}\left(\xi_{0}\right)\right|^{-1} \int_{B_{\rho}\left(\xi_{0}\right)}|X w|^{2}+\sigma^{-m+2}|X w|^{m} d \xi \\
& =\sigma^{2}\left[\left|B_{\rho}\left(\xi_{0}\right)\right|^{-1} \int_{B_{\rho}\left(\xi_{0}\right)}\left(\left|X u-p_{0}\right|^{2}+\left|X u-p_{0}\right|^{m}\right) d \xi\right] \\
& \quad \leq \frac{1}{C_{1}^{2} \Phi} \Phi \leq 1
\end{aligned}
$$

Noting (4.10) and (4.11) yields

$$
\omega^{1 / m}\left(\left|p_{0}\right|, \Phi^{1 / m}\left(\xi_{0}, \rho, p_{0}\right)\right)+\Phi^{1 / 2}\left(\xi_{0}, \rho, p_{0}\right) \leq \frac{\delta}{2} .
$$

So (4.17) and (4.18) imply the conditions (3.2) and (3.3) in Lemma 3.1, respectively. Also (1.1) and (1.3) suggest the condition (3.1). Then there exists an $\mathcal{A}$-harmonic function $h \in H W^{1, m}\left(B_{\rho}\left(\xi_{0}\right), \mathbb{R}^{N}\right)$ satisfying

$$
\left|B_{\rho}\left(\xi_{0}\right)\right|^{-1} \int_{B_{\rho}\left(\xi_{0}\right)}|X h|^{2} d \xi \leq 1
$$

and

$$
\left|B_{\rho}\left(\xi_{0}\right)\right|^{-1} \rho^{-2} \int_{B_{\rho}\left(\xi_{0}\right)}|w-h|^{2} d \xi \leq \varepsilon .
$$

Taking $u_{0}=u_{\xi_{0}, 2 \theta \rho}, \theta \in\left(0, \frac{1}{8}\right]$, and replacing $p_{0}$ by $p_{0}+\sigma^{-1}(X h)_{\xi_{0}, 2 \theta \rho}$ in Lemma 3.2 yields

$$
\begin{aligned}
& \int_{B_{\theta \rho}\left(\xi_{0}\right)}\left|X u-p_{0}-\sigma^{-1}(X h)_{\xi_{0}, 2 \theta \rho}\right|^{2} d \xi \\
& \quad+\int_{B_{\theta \rho}\left(\xi_{0}\right)}\left|X u-p_{0}-\sigma^{-1}(X h)_{\xi_{0}, 2 \theta \rho}\right|^{m} d \xi \\
& \leq \frac{C}{(\theta \rho)^{2}} \int_{B_{2 \theta \rho}\left(\xi_{0}\right)}\left|u-u_{\xi_{0}, 2 \theta \rho}-\left(p_{0}+\sigma^{-1}(X h)_{\xi_{0}, 2 \theta \rho}\right)\left(\xi^{1}-\xi_{0}^{1}\right)\right|^{2} d \xi \\
&+\frac{C}{(\theta \rho)^{m}} \int_{B_{2 \theta \rho}\left(\xi_{0}\right)}\left|u-u \xi_{0}, 2 \theta \rho-\left(p_{0}+\sigma^{-1}(X h)_{\xi_{0}, 2 \theta \rho}\right)\left(\xi^{1}-\xi_{0}^{1}\right)\right|^{m} d \xi+C\left|B_{2 \theta \rho}\left(\xi_{0}\right)\right| \\
& \times\left\{\left[K\left(\left|u_{\xi_{0}, 2 \theta \rho}\right|+\left|p_{0}+\sigma^{-1} X h\left(\xi_{0}\right)\right|\right)\left(1+\left|p_{0}+\sigma^{-1}(X h)_{\xi_{0}, 2 \theta \rho}\right|^{2}\right)^{m / 2}\right]^{2 /(1-\gamma)}(2 \theta \rho)^{2 \gamma}\right. \\
&\left.+\left(b^{2}+a^{2}\left|p_{0}+\sigma^{-1}(X h)_{\xi_{0}, 2 \theta \rho}\right|^{2 m}\right)(2 \theta \rho)^{2}\right\} \\
&:= \mathrm{I}^{\prime \prime}+\mathrm{II}^{\prime \prime}+\mathrm{III}^{\prime \prime} .
\end{aligned}
$$

Note the facts that

$$
\begin{aligned}
& \left|\sigma^{-1}(X h)_{\xi_{0}, 2 \theta \rho}\right| \\
& \quad=C_{1}\left[\Phi\left(\xi_{0}, \rho, p_{0}\right)+4 \delta^{-2} \rho^{2 \gamma} H^{2}\left(\left|u_{\xi_{0}, \rho}\right|+\left|p_{0}\right|\right)\right]^{1 / 2} \sup _{B_{2 \theta \rho}\left(\xi_{0}\right)}|X h| \\
& \quad \leq C_{1} \sqrt{C_{0}}\left[\Phi\left(\xi_{0}, \rho, p_{0}\right)+4 \delta^{-2} \rho^{2 \gamma} H^{2}\left(\left|u_{\xi_{0}, \rho}\right|+\left|p_{0}\right|\right)\right]^{1 / 2},
\end{aligned}
$$


where we have used (3.8) and (4.20), and

$$
\begin{aligned}
\mid u \xi_{0}, 2 \theta \rho & \leq \mid u \xi_{0}, \rho \\
& =\mid u \xi_{\xi_{0}, 2 \theta \rho}-u \xi_{0}, \rho \\
& =\left|u \xi_{0}, \rho\right|+\left.|| B_{2 \theta \rho}\left(\xi_{0}\right)\right|^{-1} \int_{B_{2 \theta \rho}\left(\xi_{0}\right)}\left[u-p_{0}\left(\xi^{1}-\xi_{0}^{1}\right)-u \xi_{0}, \rho\right] d \xi \mid \\
& \leq\left|u \xi_{0}, \rho\right|+\left|B_{2 \theta \rho}\left(\xi_{0}\right)\right|^{-1} \int_{B_{\rho}\left(\xi_{0}\right)} \mid u-p_{0}\left(\xi^{1}-\xi_{0}^{1}\right)-u \xi_{0}, \rho \\
& \mid d \xi \\
& \leq\left|u_{\xi_{0}, \rho}\right|+\left((2 \theta)^{-Q_{\mid}}\left|B_{\rho}\left(\xi_{0}\right)\right|^{-1} \int_{B_{\rho}\left(\xi_{0}\right)}\left|u-p_{0}\left(\xi^{1}-\xi_{0}^{1}\right)-u \xi_{0}, \rho\right|^{2} d \xi\right)^{1 / 2} \\
& \leq\left|u_{\xi_{0}, \rho}\right|+(2 \theta)^{-Q / 2} \sqrt{C_{P}} \rho \Phi^{1 / 2}\left(\xi_{0}, \rho, p_{0}\right),
\end{aligned}
$$

where we have used the fact that $u-p_{0}\left(\xi^{1}-\xi_{0}^{1}\right)$ has the mean value $u_{\xi_{0}, 2 \theta \rho}$ on the ball $B_{2 \theta \rho}\left(\xi_{0}\right)$.

It follows that by (4.23) and (4.24)

$$
\begin{aligned}
& \left|u_{\xi_{0}, 2 \theta \rho}\right|+\left|p_{0}+\sigma^{-1}(X h)_{\xi_{0}, 2 \theta \rho}\right| \\
& \leq\left|u_{\xi_{0}, \rho}\right|+\left|p_{0}\right|+\left[(2 \theta)^{-Q / 2} \sqrt{C_{P}}+C_{1} \sqrt{C_{0}}\right] \\
& \quad \times\left[\Phi\left(\xi_{0}, \rho, p_{0}\right)+4 \delta^{-2} \rho^{2 \gamma} H^{2}\left(\left|u_{\xi_{0}, \rho}\right|+\left|p_{0}\right|\right)\right]^{1 / 2} \\
& \leq\left|u_{\xi_{0}, \rho}\right|+\left|p_{0}\right|+C_{2} \Phi^{1 / 2}\left(\xi_{0}, \rho, p_{0}\right)+2 \delta^{-1} \rho^{\gamma} C_{2} H\left(\left|u_{\xi_{0}, \rho}\right|+\left|p_{0}\right|\right) .
\end{aligned}
$$

On the other hand, we see from (4.11) and (4.12) that

$$
\left[(2 \theta)^{-Q / 2} \sqrt{C_{P}}+C_{1} \sqrt{C_{0}}\right] \Phi^{1 / 2}\left(\xi_{0}, \rho, p_{0}\right) \leq 1 / 2
$$

and

$$
2 \delta^{-1} \rho^{\gamma}\left[(2 \theta)^{-Q / 2} \sqrt{C_{P}}+C_{1} \sqrt{C_{0}}\right] H\left(\left|u_{\xi_{0}, \rho}\right|+\left|p_{0}\right|\right) \leq 1 / 2
$$

So

$$
\left|u_{\xi_{0}, 2 \theta \rho}\right|+\left|p_{0}+\sigma^{-1}(X h)_{\xi_{0}, 2 \theta \rho}\right| \leq 1+\left|u_{\xi_{0}, \rho}\right|+\left|p_{0}\right| .
$$

Thus we first get,

$$
\mathrm{III}^{\prime \prime} \leq C \omega_{X}(2 \theta \rho)^{Q+2 \gamma} H^{2}\left(1+\left|u_{\xi_{0}, \rho}\right|+\left|p_{0}\right|\right) .
$$

Next, we recall that $g(\tau)=\int_{B_{2 \theta \rho}\left(\xi_{0}\right)}(u-\tau)^{2} d \xi$ has a minimal value at $\tau=u_{\xi_{0}, 2 \theta \rho}$, and $u-\left(p_{0}+\sigma^{-1}(X h)_{\xi_{0}, 2 \theta \rho}\right)\left(\xi^{1}-\xi_{0}^{1}\right)$ has the mean value $u_{\xi_{0}, 2 \theta \rho}$ on $B_{2 \theta \rho}\left(\xi_{0}\right)$. Using (4.20) and (2.6) yields

$$
\begin{aligned}
&\left|B_{2 \theta \rho}\left(\xi_{0}\right)\right|^{-1}(2 \theta \rho)^{-2} \int_{B_{2 \theta \rho\left(\xi_{0}\right)}}\left|u(\xi)-u_{\xi_{0}, 2 \theta \rho}-\left(p_{0}+\sigma^{-1}(X h)_{\xi_{0}, 2 \theta \rho}\right)\left(\xi^{1}-\xi_{0}^{1}\right)\right|^{2} d \xi \\
& \leq \sigma^{-2}\left|B_{2 \theta \rho}\left(\xi_{0}\right)\right|^{-1}(2 \theta \rho)^{-2} \int_{B_{2 \theta \rho\left(\xi_{0}\right)}}\left|w(\xi)-h_{\xi_{0}, 2 \theta \rho}-(X h)_{\xi_{0}, 2 \theta \rho}\left(\xi^{1}-\xi_{0}^{1}\right)\right|^{2} d \xi \\
& \leq 2 \sigma^{-2}\left|B_{2 \theta \rho}\left(\xi_{0}\right)\right|^{-1}(2 \theta \rho)^{-2} \\
& \quad \times \int_{B_{2 \theta \rho\left(\xi_{0}\right)}}\left[|w(\xi)-h(\xi)|^{2}+\left|h(\xi)-h_{\xi_{0}, 2 \theta \rho}-(X h)_{\xi_{0}, 2 \theta \rho}\left(\xi^{1}-\xi_{0}^{1}\right)\right|^{2}\right] d \xi
\end{aligned}
$$




$$
\begin{aligned}
& \leq 2 \sigma^{-2}\left[(2 \theta)^{-Q-2} \varepsilon+C_{P}\left|B_{2 \theta \rho}\left(\xi_{0}\right)\right|^{-1} \int_{B_{2 \theta \rho\left(\xi_{0}\right)}}\left|X h(\xi)-(X h)_{\xi_{0}, 2 \theta \rho}\right|^{2} d \xi\right] \\
& \leq 2 \sigma^{-2}\left[(2 \theta)^{-Q-2} \varepsilon+C_{P}^{2}(2 \theta \rho)^{2}\left|B_{2 \theta \rho}\left(\xi_{0}\right)\right|^{-1} \int_{B_{2 \theta \rho\left(\xi_{0}\right)}}\left|X^{2} h(\xi)\right|^{2} d \xi\right] .
\end{aligned}
$$

By (3.7) with $k=2$, the inequality

$$
\int_{B_{R / 4}\left(\xi_{0}\right)}\left|X^{2} h\right|^{2} d \xi \leq C R^{-4} \int_{B_{R}\left(\xi_{0}\right)}|h|^{2} d \xi
$$

is valid. Replacing $h$ with $h-h_{\xi_{0}, R}$ and using (2.6) leads to

$$
\int_{B_{R / 4}\left(\xi_{0}\right)}\left|X^{2} h\right|^{2} d \xi \leq C R^{-4} \int_{B_{R}\left(\xi_{0}\right)}\left|h-h_{\xi_{0}, R}\right|^{2} d \xi \leq C C_{P} R^{-2} \int_{B_{R}\left(\xi_{0}\right)}|X h|^{2} d \xi .
$$

It implies that

$$
\sup _{B_{R / 4}\left(\xi_{0}\right)}\left|X^{2} h\right|^{2} \leq 2^{2 Q_{C}} C C_{P} R^{-2}\left|B_{R}\left(\xi_{0}\right)\right|^{-1} \int_{B_{R}\left(\xi_{0}\right)}|X h|^{2} d \xi .
$$

Applying (4.20), it follows that for $\theta \in\left(0, \frac{1}{8}\right]$

$$
\begin{aligned}
& \left|B_{2 \theta \rho}\left(\xi_{0}\right)\right|^{-1} \int_{B_{2 \theta \rho}\left(\xi_{0}\right)}\left|X^{2} h(\xi)\right|^{2} d \xi \\
& \leq \sup _{B_{2 \theta \rho}\left(\xi_{0}\right)}\left|X^{2} h(\xi)\right|^{2} \leq \sup _{B_{\rho / 4}\left(\xi_{0}\right)}\left|X^{2} h(\xi)\right|^{2} \\
& \quad \leq 2^{2 Q} C C_{P} \rho^{-2}\left|B_{\rho}\left(\xi_{0}\right)\right|^{-1} \int_{B_{\rho}\left(\xi_{0}\right)}|X h|^{2} d \xi \leq 2^{2 Q_{C}} C C_{P} \rho^{-2} .
\end{aligned}
$$

Noting that $\sigma=C_{1}^{-1}\left[\Phi\left(\xi_{0}, \rho, p_{0}\right)+4 \delta^{-2} \rho^{2 \gamma} H^{2}\left(\left|u_{0}\right|+\left|p_{0}\right|\right)\right]^{-1 / 2}$, we estimate the left-hand side of (4.28):

$$
\begin{aligned}
& \leq 2 \sigma^{-2}\left[(2 \theta)^{-Q-2} \varepsilon+C_{P}^{2}(2 \theta \rho)^{2}\left|B_{2 \theta \rho}\left(\xi_{0}\right)\right|^{-1} \int_{B_{2 \theta \rho\left(\xi_{0}\right)}}\left|X^{2} h(\xi)\right|^{2} d \xi\right] \\
& \leq \sigma^{-2}\left[2^{-Q-1} \theta^{-Q-2} \varepsilon+2^{2 Q+3} C C_{P}^{3} \theta^{2}\right] \\
& \leq C_{4}\left[\theta^{-Q-2} \varepsilon+\theta^{2}\right]\left[\Phi\left(\xi_{0}, \rho, p_{0}\right)+4 \delta^{-2} \rho^{2 \gamma} H^{2}\left(\left|u_{\xi_{0}, \rho}\right|+\left|p_{0}\right|\right)\right],
\end{aligned}
$$

where $C_{4}=C_{1}^{2}\left(2^{-Q-1}+2^{2 Q+3} C C_{P}^{3}\right)>1$.

Thus it follows that

$$
\mathrm{I}^{\prime \prime} \leq 4 C_{4} C \| B_{2 \theta \rho}\left(\xi_{0}\right) \mid\left[(\theta)^{-Q-2} \varepsilon+\theta^{2}\right]\left[\Phi\left(\xi_{0}, \rho, p_{0}\right)+4 \delta^{-2} \rho^{2 \gamma} H^{2}\left(\left\|u_{\xi_{0}, \rho}\left|+\| p_{0}\right|\right)\right] .\right.
$$

For $2<m<Q$, we have $(Q-m) / Q m=1 / m^{*}<1 / m<\frac{1}{2}$. Then there exists $t \in[0,1)$ such that $1 / m=\frac{1}{2}(1-t)+\left(1 / m^{*}\right) t$. Using (2.9), (2.12), Young's inequality and (4.29) gives

$$
\begin{aligned}
& \int_{B_{2 \theta \rho}\left(\xi_{0}\right)}\left|u-u \xi_{\xi_{0}, 2 \theta \rho}-\left(p_{0}+\sigma^{-1}(X h)_{\xi_{0}, 2 \theta \rho}\right)\left(\xi^{1}-\xi_{0}^{1}\right)\right|^{m} d \xi \\
& \leq\left[\int_{B_{2 \theta \rho}\left(\xi_{0}\right)}\left|u-u \xi_{\xi_{0}, 2 \theta \rho}-\left(p_{0}+\sigma^{-1}(X h)_{\xi_{0}, 2 \theta \rho}\right)\left(\xi^{1}-\xi_{0}^{1}\right)\right|^{2} d \xi\right]^{(1-t) m / 2} \\
& \quad \times\left[\int_{B_{2 \theta \rho}\left(\xi_{0}\right)}\left|u-u \xi_{0}, 2 \theta \rho-\left(p_{0}+\sigma^{-1}(X h)_{\xi_{0}, 2 \theta \rho}\right)\left(\xi^{1}-\xi_{0}^{1}\right)\right|^{m^{*}} d \xi\right]^{t m / m^{*}}
\end{aligned}
$$




$$
\begin{aligned}
\leq & S^{t m}\left\{\left|B_{2 \theta \rho}\left(\xi_{0}\right)\right|(2 \theta \rho)^{2} C_{4}\left[(\theta)^{-Q-2} \varepsilon+\theta^{2}\right]\right. \\
& \left.\times\left[\Phi\left(\xi_{0}, \rho, p_{0}\right)+4 \delta^{-2} \rho^{2 \gamma} H^{2}\left(\left|u_{\xi_{0}, \rho}\right|+\left|p_{0}\right|\right)\right]\right\}^{(1-t) m / 2} \\
& \times\left(\int_{B_{2 \theta \rho}\left(\xi_{0}\right)}\left|X u-\left(p_{0}+\sigma^{-1}(X h)_{\xi_{0}, 2 \theta \rho}\right)\right|^{m} d \xi\right)^{t} \\
\leq & \left(S^{t m} \varepsilon^{-t}\right)^{1 /(1-t)}\left\{\left|B_{2 \theta \rho}\left(\xi_{0}\right)\right|(2 \theta \rho)^{2} C_{4}\left[(\theta)^{-Q-2} \varepsilon+\theta^{2}\right]\right. \\
& \left.\times\left[\Phi\left(\xi_{0}, \rho, p_{0}\right)+4 \delta^{-2} \rho^{2 \gamma} H^{2}\left(\left|u_{\xi_{0}, \rho}\right|+\left|p_{0}\right|\right)\right]\right\}^{m / 2} \\
& +\varepsilon \int_{B_{2 \theta \rho}\left(\xi_{0}\right)}\left|X u-\left(p_{0}+\sigma^{-1}(X h)_{\xi_{0}, 2 \theta \rho}\right)\right|^{m} d \xi \\
\leq & \left(S^{t m} \varepsilon^{-t}\right)^{1 /(1-t)}\left(\left|B_{2 \theta \rho}\left(\xi_{0}\right)\right|(2 \theta \rho)^{2} C_{4}\right)^{m / 2}\left[(\theta)^{-Q-2} \varepsilon+\theta^{2}\right] \\
& \times\left[\Phi\left(\xi_{0}, \rho, p_{0}\right)+4 \delta^{-2} \rho^{2 \gamma} H^{2}\left(\left|u_{\xi_{0}, \rho}\right|+\left|p_{0}\right|\right)\right] \\
& +\varepsilon \int_{B_{2 \theta \rho}\left(\xi_{0}\right)}\left|X u-\left(p_{0}+\sigma^{-1}(X h)_{\xi_{0}, 2 \theta \rho}\right)\right|^{m} d \xi .
\end{aligned}
$$

Thus we obtain

$$
\begin{aligned}
\mathrm{II}^{\prime \prime} \leq & C\left(S^{t m} \varepsilon^{-t}\right)^{1 /(1-t)}\left(4 C_{4}\right)^{m / 2}\left|B_{2 \theta \rho}\left(\xi_{0}\right)\right|^{m / 2}\left[(\theta)^{-Q-2} \varepsilon+\theta^{2}\right] \\
& \times\left[\Phi\left(\xi_{0}, \rho, p_{0}\right)+4 \delta^{-2} \rho^{2 \gamma} H^{2}\left(\mid u \xi_{0}, \rho\right.\right. \\
& \left.\left.|+| p_{0} \mid\right)\right] \\
& +C \varepsilon \int_{B_{2 \theta \rho}\left(\xi_{0}\right)}\left|X u-\left(p_{0}+\sigma^{-1}(X h)_{\xi_{0}, 2 \theta \rho}\right)\right|^{m} d \xi
\end{aligned}
$$

Substituting these estimates of I", II", III" into (4.22), we have

$$
\begin{aligned}
& \int_{B_{\theta \rho}\left(\xi_{0}\right)}\left|X u-p_{0}-\sigma^{-1}(X h)_{\xi_{0}, 2 \theta \rho}\right|^{2} d \xi \\
& \quad+(1-C \varepsilon) \int_{B_{\theta \rho}\left(\xi_{0}\right)}\left|X u-\left(p_{0}+\sigma^{-1}(X h)_{\xi_{0}, 2 \theta \rho}\right)\right|^{m} d \xi \\
& \leq C\left(4 C_{4}\right)^{m / 2}\left[\left(S^{t m} \varepsilon^{-t}\right)^{1 /(1-t)}+1\right]\left[(\theta)^{-Q-2} \varepsilon+\theta^{2}\right]\left|B_{2 \theta \rho}\left(\xi_{0}\right)\right| \\
& \times\left[\Phi\left(\xi_{0}, \rho, p_{0}\right)+4 \delta^{-2} \rho^{2 \gamma} H^{2}\left(\left|u \xi_{0}, \rho\right|+\left|p_{0}\right|\right)\right] \\
&+C \omega_{X}(2 \theta \rho)^{Q+2 \gamma} H^{2}\left(1+\left|u_{\xi_{0}, \rho}\right|+\left|p_{0}\right|\right) \\
&:= C_{5}\left[(\theta)^{-Q-2} \varepsilon+\theta^{2}\right]\left|B_{2 \theta \rho}\left(\xi_{0}\right)\right|\left[\Phi\left(\xi_{0}, \rho, p_{0}\right)+4 \delta^{-2} \rho^{2 \gamma} H^{2}\left(\left|u_{\xi_{0}, \rho}\right|+\left|p_{0}\right|\right)\right] \\
&+C \omega_{X}(2 \theta \rho)^{Q+2 \gamma} H^{2}\left(1+\left|u_{\xi_{0}, \rho}\right|+\left|p_{0}\right|\right),
\end{aligned}
$$

where $C_{5}=C\left(4 C_{4}\right)^{m / 2}\left[\left(S^{t m} \varepsilon^{-t}\right)^{1 /(1-t)}+1\right]$. Then it follows that

$\Phi\left(\xi_{0}, \theta \rho,(X u)_{\xi_{0}, \theta \rho}\right)$

$$
\begin{aligned}
& =\left|B_{\theta \rho}\left(\xi_{0}\right)\right|^{-1} \int_{B_{\theta \rho}\left(\xi_{0}\right)}\left[\left|X u-(X u)_{\xi_{0}, \theta \rho}\right|^{2}+\left|X u-(X u)_{\xi_{0}, \theta \rho}\right|^{m}\right] d \xi \\
& \leq\left|B_{\theta \rho}\left(\xi_{0}\right)\right|^{-1} \int_{B_{\theta \rho}\left(\xi_{0}\right)}\left[\left|X u-p_{0}-\sigma^{-1}(X h)_{\xi_{0}, 2 \theta \rho}\right|^{2}+\left|X u-p_{0}-\sigma^{-1}(X h)_{\xi_{0}, 2 \theta \rho}\right|^{m}\right] d \xi
\end{aligned}
$$




$$
\begin{aligned}
\leq & C_{5} \frac{2^{Q}\left(\theta^{-Q-2} \varepsilon+\theta^{2}\right)}{(1-C \varepsilon)}\left[\Phi\left(\xi_{0}, \rho, p_{0}\right)+4 \delta^{-2} \rho^{2 \gamma} H^{2}\left(1+\left|u_{\xi_{0}, \rho}\right|+\left|p_{0}\right|\right)\right] \\
& +C \omega_{X}(2 \theta \rho)^{2 \gamma} 2^{Q} H^{2}\left(1+\left|u_{\xi_{0}, \rho}\right|+\left|p_{0}\right|\right) \\
\leq & C_{6}\left(\theta^{-Q-2} \varepsilon+\theta^{2}\right) \Phi\left(\xi_{0}, \rho, p_{0}\right)+C_{3}(\theta \rho)^{2 \gamma} H^{2}\left(1+\left|u_{\xi_{0}, \rho}\right|+\left|p_{0}\right|\right),
\end{aligned}
$$

where $C_{6}=C_{5} 2^{Q} /(1-C \varepsilon)>1, C_{3}=4 C_{6} \delta^{-2}+2^{Q+2 \gamma} C \omega_{X}>1$.

For given $\gamma \in(0,1)$, fix $\theta \in\left(0, \frac{1}{4}\right]$ sufficiently small such that $C_{6} \theta^{2}<\frac{1}{4} \theta^{2 \gamma}$, and take $\varepsilon=\theta^{Q+4}$. Then

$$
\Phi\left(\xi_{0}, \theta \rho\right) \leq \theta^{2 \gamma}\left[\frac{1}{2} \Phi\left(\xi_{0}, \rho\right)+C_{3} \rho^{2 \gamma} H^{2}\left(1+\left|u_{\xi_{0}, \rho}\right|+\left|p_{0}\right|\right)\right],
$$

where $\Phi\left(\xi_{0}, \theta \rho\right)=: \Phi\left(\xi_{0}, \theta \rho,(X u)_{\xi_{0}, \theta \rho}\right)$. Applying (4.16) to (4.31), we conclude (4.13).

Remark 4.1. Actually, the conclusion is still valid when the factor $\frac{1}{2}$ is removed in two inequalities of (4.16). Here, we make the stronger restriction on purpose so we can use it later.

Third step. We claim that for every $n \in \mathbb{N}$,

$$
\left|u_{\xi_{0}, \theta^{n} \rho}\right|+\left|(X u)_{\xi_{0}, \theta^{n} \rho}\right|<M_{1}, \quad \Phi\left(\xi_{0}, \theta^{n} \rho\right)<t_{0} .
$$

It allows us to make an iteration from the assertion in the second step and have

$$
\Phi\left(\xi_{0}, \theta^{n+1} \rho\right) \leq \theta^{2 \gamma}\left(\frac{1}{2} \Phi\left(\xi_{0}, \theta^{n} \rho\right)+C_{3}\left(\theta^{n} \rho\right)^{2 \gamma} H_{0}^{2}\right) .
$$

Proof. We will show (4.32) by induction. Clearly, if $n=0$, then (4.33) is just (4.13). Assuming that (4.32) is true for $1, \ldots, n-1$, we have

$$
\begin{aligned}
\Phi\left(\xi_{0}, \theta^{n} \rho\right) & \leq \frac{\theta^{2 n \gamma}}{2^{n}} \Phi\left(\xi_{0}, \rho\right)+C_{3} \theta^{2 n \gamma} \rho^{2 \gamma} H_{0}^{2} \sum_{l=0}^{n-1}\left(\frac{1}{2}\right)^{l} \\
& \leq \theta^{2 n \gamma}\left(\frac{1}{2^{n}} \Phi\left(\xi_{0}, \rho\right)+2 C_{3} \rho^{2 \gamma} H_{0}^{2}\right) \\
& \leq \theta^{2 n \gamma} t_{0} .
\end{aligned}
$$

A direct calculation gives

$$
\begin{aligned}
& \left|u_{\xi_{0}, \theta^{n} \rho}\right| \\
& \leq\left|u_{\xi_{0}, \theta^{n-1} \rho}\right|+\left|B_{\theta^{n} \rho}\left(\xi_{0}\right)\right|^{-1} \int_{B_{\theta^{n} \rho}\left(\xi_{0}\right)}\left|u-p_{0}\left(\xi^{1}-\xi_{0}^{1}\right)-u_{\xi_{0}, \theta^{n-1} \rho}\right| d \xi \\
& \leq\left|u_{\xi_{0}, \theta^{n-1} \rho}\right|+\left[\left|B_{\theta^{n} \rho}\left(\xi_{0}\right)\right|^{-1} \int_{B_{\theta^{n} \rho}\left(\xi_{0}\right)}\left|u-p_{0}\left(\xi^{1}-\xi_{0}^{1}\right)-u_{\xi_{0}, \theta^{n-1} \rho}\right|^{2} d \xi\right]^{1 / 2} \\
& \leq\left|u_{\xi_{0}, \theta^{n-1} \rho}\right|+\theta^{-Q / 2}\left[\left|B_{\theta^{n-1} \rho}\left(\xi_{0}\right)\right|^{-1} \int_{B_{\theta^{n-1} \rho}\left(\xi_{0}\right)}\left|u-p_{0}\left(\xi^{1}-\xi_{0}^{1}\right)-u_{\xi_{0}, \theta^{n-1} \rho}\right|^{2} d \xi\right]^{1 / 2} \\
& \leq\left|u_{\xi_{0}, \theta^{n-1} \rho}\right|+\theta^{-Q / 2} \sqrt{C_{P}} \theta^{n-1} \rho\left[\left|B_{\theta^{n-1} \rho}\left(\xi_{0}\right)\right|^{-1} \int_{B_{\theta^{n-1} \rho}\left(\xi_{0}\right)}\left|X u-p_{0}\right|^{2} d \xi\right]^{1 / 2} \\
& \leq\left|u_{\xi_{0}, \theta^{n-1} \rho}\right|+\theta^{-Q / 2} \sqrt{C_{P}} \theta^{n-1} \rho \Phi^{1 / 2}\left(\xi_{0}, \theta^{n-1} \rho\right)
\end{aligned}
$$


and

$$
\begin{aligned}
& \left|(X u)_{\xi_{0}, \theta^{n} \rho}\right| \\
& \quad \leq\left|(X u)_{\xi_{0}, \theta^{n-1} \rho}\right|+\left|B_{\theta^{n} \rho}\left(\xi_{0}\right)\right|^{-1} \int_{B_{\theta^{n} \rho}\left(\xi_{0}\right)}\left|X u-(X u)_{\xi_{0}, \theta^{n-1} \rho}\right| d \xi \\
& \quad \leq\left|(X u)_{\xi_{0}, \theta^{n-1} \rho}\right|+\left[\left|B_{\theta^{n} \rho}\left(\xi_{0}\right)\right|^{-1} \int_{B_{\theta^{n} \rho}\left(\xi_{0}\right)}\left|X u-(X u)_{\xi_{0}, \theta^{n-1} \rho}\right|^{2} d \xi\right]^{1 / 2} \\
& \quad \leq\left|(X u)_{\xi_{0}, \theta^{n-1} \rho}\right|+\theta^{-Q / 2}\left[\left|B_{\theta^{n-1} \rho}\left(\xi_{0}\right)\right|^{-1} \int_{B_{\theta^{n-1} \rho}\left(\xi_{0}\right)}\left|X u-(X u)_{\xi_{0}, \theta^{n-1} \rho}\right|^{2} d \xi\right]^{1 / 2} \\
& \quad \leq\left|(X u)_{\xi_{0}, \theta^{n-1} \rho}\right|+\theta^{-Q / 2} \Phi^{1 / 2}\left(\xi_{0}, \theta^{n-1} \rho\right) .
\end{aligned}
$$

Therefore one has

$$
\begin{aligned}
& \left|u_{\xi_{0}, \theta^{n} \rho}\right|+\left|(X u)_{\xi_{0}, \theta^{n} \rho}\right| \\
& \quad \leq\left|u_{\xi_{0}, \rho}\right|+\left|(X u)_{\xi_{0}, \rho}\right|+\left(1+\sqrt{C_{P}}\right) \theta^{-Q / 2} \sum_{l=0}^{n-1} \Phi^{1 / 2}\left(\xi_{0}, \theta^{l} \rho\right) \\
& \quad \leq \frac{1}{2} M_{1}+\left(1+\sqrt{C_{P}}\right) \theta^{-Q / 2} \sum_{l=0}^{n-1} \theta^{l \gamma} \sqrt{t_{0}} \\
& \quad \leq \frac{1}{2} M_{1}+\left(1+\sqrt{C_{P}}\right) \theta^{-Q / 2}\left(1-\theta^{\gamma}\right)^{-1} \sqrt{t_{0}} \leq M_{1},
\end{aligned}
$$

where we have used (4.16), (4.34) and (4.14), respectively.

Now, combining (4.34) and (4.35) implies (4.32) for all $n \in \mathbb{N}$.

Fourth step. We claim that $u \in \Gamma^{1, \gamma}\left(\Omega_{0}, \mathbb{R}^{N}\right)$, where $\Omega_{0} \subset \Omega$ is an open subset such that $\operatorname{meas}\left(\Omega \backslash \Omega_{0}\right)=0$.

Proof. We have from the third step that

$$
\Phi\left(\xi_{0}, \theta^{n} \rho\right) \leq \theta^{2 n \gamma} t_{0}, \quad \text { for } n \in \mathbb{N} .
$$

Since for every $\rho \in\left(0, \rho_{0}\right]$ there exists a positive constant $n$ such that

$$
\theta^{n+1} \rho_{0}<\rho \leq \theta^{n} \rho_{0},
$$

it follows that for given $\theta$ and $\rho_{0}$,

$$
\begin{aligned}
\Phi\left(\xi_{0}, \rho\right) & =\left|B_{\rho}\left(\xi_{0}\right)\right|^{-1} \int_{B_{\rho}\left(\xi_{0}\right)}\left|X u-p_{0}\right|^{2}+\left|X u-p_{0}\right|^{m} d \xi \\
& \leq\left|B_{\theta^{n+1} \rho_{0}}\left(\xi_{0}\right)\right|^{-1} \int_{B_{\theta^{n} \rho_{0}}\left(\xi_{0}\right)}\left|X u-p_{0}\right|^{2}+\left|X u-p_{0}\right|^{m} d \xi \\
& \leq C\left|B_{\theta^{n} \rho_{0}}\left(\xi_{0}\right)\right|^{-1} \int_{B_{\theta^{n} \rho_{0}}\left(\xi_{0}\right)}\left|X u-p_{0}\right|^{2}+\left|X u-p_{0}\right|^{m} d \xi \\
& =C \Phi\left(\xi_{0}, \theta^{n} \rho_{0}\right) \leq C \theta^{2 n \gamma} t_{0} \leq C\left(\theta^{-1} \frac{\rho}{\rho_{0}}\right)^{2 \gamma} \leq C \rho^{2 \gamma}
\end{aligned}
$$


Observing that (4.16) still holds for any $\xi$ sufficiently close to $\xi_{0}$, then there exists a ball $B_{r}\left(\xi_{0}\right)$ centered at $\xi_{0}$ such that for any $\xi \in B_{r}\left(\xi_{0}\right)$,

$$
\Phi(\xi, \rho) \leq C \rho^{2 \gamma} .
$$

So we infer that $X u \in \mathcal{L}^{2, \mu}\left(B_{\rho}\left(\xi_{0}\right), \mathbb{R}^{N}\right)$ with $\mu=Q+2 \gamma$. Using Lemma 2.6 implies $X u \in \Gamma^{\gamma}\left(B_{\rho}\left(\xi_{0}\right), \mathbb{R}^{N}\right)$.

Noting the facts that $u \in H W^{1, m}\left(\Omega, \mathbb{R}^{N}\right) \cap L^{\infty}\left(\Omega, \mathbb{R}^{N}\right)$ and the continuity of $\Phi\left(\xi_{0}, \rho\right)$, we conclude that meas $\left(\Omega \backslash \Omega_{0}\right)=\operatorname{meas}\left(\Sigma_{1} \cup \Sigma_{2}\right)=0$.

Proof of Theorem 1.2. Similar to (3.13), we have

$$
\begin{aligned}
& \int_{B_{R}\left(\xi_{0}\right)}\left[A_{i}^{\alpha}(\xi, u, X u)-A_{i}^{\alpha}\left(\xi, u, p_{0}\right)\right] \eta^{2}\left(X u-p_{0}\right) d \xi \\
&=-2 \int_{B_{R}\left(\xi_{0}\right)}\left[A_{i}^{\alpha}(\xi, u, X u)-A_{i}^{\alpha}\left(\xi, u, p_{0}\right)\right] \eta v X \eta d \xi \\
&-\int_{B_{R}\left(\xi_{0}\right)} A_{i}^{\alpha}\left(\xi, u, p_{0}\right) X \varphi^{\alpha} d \xi \\
& \leq 2 C \int_{B_{R}\left(\xi_{0}\right)}\left(1+|X u|^{2}+\left|p_{0}\right|^{2}\right)^{(m-2) / 2}\left|X u-p_{0}\right||\eta||v||X \eta| d \xi \\
&+K(\cdot) \int_{B_{R}\left(\xi_{0}\right)}\left|u-u_{0}-\left(\xi^{1}-\xi_{0}^{1}\right) p_{0}\right|^{\gamma}\left(1+\left|p_{0}\right|\right)^{m / 2} \eta^{2}|X v| d \xi \\
&+2 K(\cdot) \int_{B_{R}\left(\xi_{0}\right)}\left|u-u_{0}-\left(\xi^{1}-\xi_{0}^{1}\right) p_{0}\right|^{\gamma}\left(1+\left|p_{0}\right|\right)^{m / 2}|\eta||v||X \eta| d \xi \\
&+K(\cdot) \int_{B_{R}\left(\xi_{0}\right)}\left[\left(\left|\left(\xi_{0}\right)^{-1} \cdot \xi\right|^{m}+\left|\left(\xi^{1}-\xi_{0}^{1}\right) p_{0}\right|^{m}\right)^{\gamma / m}\left(1+\left|p_{0}\right|\right)^{m / 2}\right. \\
&\left.\times\left(\eta^{2}|X v|+2|\eta||v||X \eta|\right)\right] d \xi \\
&+\int_{B_{R}\left(\xi_{0}\right)}\left(a|X u|^{m-\epsilon}+b\right)|v|^{2} d \xi \\
&:= \mathrm{I}+\mathrm{II}+\mathrm{III}+\mathrm{IV}+\mathrm{V}^{\prime},
\end{aligned}
$$

where I, II, III, IV are terms in (3.13).

Applying Hölder's inequality, Young's inequality and noting that $\sup _{\Omega}|u|=M$, we find

$$
\begin{aligned}
V^{\prime} \leq & \int_{B_{R}\left(\xi_{0}\right)}\left(a|X u|^{m-\varepsilon}+b\right)|v| \eta^{2} d \xi \\
\leq & a\left[\int_{B_{R}\left(\xi_{0}\right)}|X u|^{m} \eta^{2 m /(m-\varepsilon)} d \xi\right]^{(m-\varepsilon) / m}\left[\int_{B_{R}\left(\xi_{0}\right)}|v|^{m / \varepsilon} d \xi\right]^{\varepsilon / m}+b \int_{B_{R}\left(\xi_{0}\right)}|v| \eta^{2} d \xi \\
\leq & a\left(2 M+R\left|p_{0}\right|\right)^{1-\varepsilon}\left[\int_{B_{R}\left(\xi_{0}\right)}|X u|^{m} \eta^{2} d \xi\right]^{(m-\varepsilon) / m}\left[\int_{B_{R}\left(\xi_{0}\right)}|v|^{m} d \xi\right]^{\varepsilon / m} \\
& +b \int_{B_{R}\left(\xi_{0}\right)}|v| \eta^{2}\left(\frac{R}{R-\rho}\right) d \xi
\end{aligned}
$$




$$
\begin{aligned}
\leq & 2^{m-1} a\left(2 M+R\left|p_{0}\right|\right)^{1-\varepsilon} \varepsilon^{\prime} \int_{B_{R}\left(\xi_{0}\right)}\left|X u-p_{0}\right|^{m} \eta^{2} d \xi \\
& +2^{m-1} a\left(2 M+R\left|p_{0}\right|\right)^{1-\varepsilon} \varepsilon^{\prime}\left|p_{0}\right|^{m}\left|B_{R}\left(\xi_{0}\right)\right| \\
& +\varepsilon^{-(m-\varepsilon) / \varepsilon} a\left(2 M+R\left|p_{0}\right|\right)^{1-\varepsilon}(R-\rho)^{-m} \int_{B_{R}\left(\xi_{0}\right)}|v|^{m} d \xi \\
& +\varepsilon^{\prime-1}(R-\rho)^{-2} \int_{B_{R}\left(\xi_{0}\right)}|v|^{2} d \xi+b^{2} R^{2}\left|B_{R}\left(\xi_{0}\right)\right| \varepsilon^{\prime} .
\end{aligned}
$$

Then it is not difficult to obtain a Caccioppoli-type inequality like Lemma 3.2. Finally, we end the proof of Theorem 1.2 by following the process of the proof of Theorem 1.1.

Acknowledgements. We would like to express our gratitude to anonymous referees for valuable suggestions. This project was supported by the Natural Science Foundation of Jiangxi, China, No. 2010GQS0021, and supported by Science and Technology Planning Project of Jiangxi Province, China, No. GJJ11219.

\section{REFERENCES}

[1] M. Bramanti and L. Brandolini. $L^{p}$-estimates for nonvariational hypoelliptic operators with VMO coefficients. Trans. Amer. Math. Soc. 352 (2000), 781-822.

[2] M. Bramanti and L. Brandolini. Schauder estimates for parabolic nondivergence operators of Hörmander type. J. Differential Equations 234 (2007), 177-245.

[3] L. Capogna. Regularity of quasi-linear equations in the Heisenberg group. Comm. Pure Appl. Math. 50 (1997), 867-889.

[4] L. Capogna. Regularity for quasilinear equations and 1-quasiconformal maps in Carnot groups. Math. Ann. 313 (1999), 263-295.

[5] L. Capogna, D. Danielli and N. Garofalo. An embedding theorem and the Harnack inequality for nonlinear subelliptic equations. Comm. Partial Differential Equations 18 (1993), 1765-1794.

[6] S. Chen and Z. Tan. Optimal interior partial regularity for nonlinear elliptic systems under the natural growth condition: the method of A-harmonic approximation. Acta Math. Sci. 27 (2007), 491-508.

[7] S. Chen and Z. Tan. The method of A-harmonic approximation and optimal interior partial regularity for nonlinear elliptic systems under the controllable growth condition. J. Math. Anal. Appl. 335 (2007), 20-42.

[8] A. Domokos. Differentiability of solutions for the non-degenerate $p$-Laplacian in the Heisenberg group. J. Differential Equations 204 (2004), 439-470.

[9] A. Domokos. On the regularity of $p$-harmonic functions in the Heisenberg group. PhD Thesis, University of Pittsburgh, 2004.

[10] F. Duzaar and G. Mingione. The $p$-harmonic approximation and the regularity of $p$-harmonic maps. Calc. Var. Partial Differential Equations 20 (2004), 235-256.

[11] F. Duzaar and G. Mingione. Regularity for degenerate elliptic problems via $p$-harmonic approximation. Ann. Inst. H. Poincaré Anal. Non Linèaire 21 (2004), 735-766.

[12] F. Duzaar and J. F. Grotowski. Partial regularity for nonlinear elliptic systems: the method of A-harmonic approximation. Manuscripta Math. 103 (2000), 267-298.

[13] A. Föglein. Partial regularity results for subelliptic systems in the Heisenberg group. Calc. Var. Partial Differential Equations 32 (2008), 25-51.

[14] N. Garofalo. Gradient bounds for the horizontal $p$-Laplacian on a Carnot group and some applications. Manuscripta Mathematica 130 (2009), 375-385.

[15] M. Giaquinta and G. Modica. Partial regularity of minimizers of quasiconvex integrals. Ann. Inst. H. Poincaré 3 (1986), 185-208.

[16] D. F. Giuseppe and S. F. Maria. Gradient estimates for elliptic systems in Carnot-Carathéodory spaces. Comment. Math. Univ. Carolinae 43 (2002), 605-618.

[17] N. Garofalo and D. M. Nhieu. Isoperimetric and Sobolev inequalities for Carnot-Carathéodory spaces and the existence of minimal surfaces. Commun. Pure Appl. Math. 49 (1996), 1081-1144. 
[18] L. Hörmander. Hypoelliptic second order differential equation. Acta Math. Uppsala 119 (1967), 147-171.

[19] D. Jerison. The Poincaré inequality for vector fields satisfying Hörmander's condition. Duke Math. J. 53 (1986), 503-523.

[20] G. Lu. Embedding theorem on Campanato-Morrey spaces for vector fields and applications. C. R. Acad. Sci. Paris 320 (1995), 429-434.

[21] G. Lu. Embedding theorems into Lipschitz and BMO spaces and applications to quasilinear subelliptic differential equations. Publ. Mat. 40 (1996), 310-329.

[22] S. Marchi. $C^{1, \alpha}$ local regularity for the solutions of the $p$-Laplacian on the Heisenberg group for $2<p<$ $1+\sqrt{5}$. Z. Anal. Anwend. 20 (2001), 617-636.

[23] S. Marchi. $C^{1, \alpha}$ local regularity for the solutions of the $p$-Laplacian on the Heisenberg group for $1+\frac{1}{\sqrt{5}}<$ $p \leq 2$. Comm. Math. Univ. Carolinae 44 (2003), 33-56.

[24] J. Manfredi and G. Mingione. Regularity results for quasilinear elliptic equations in the Heisenberg group. Math. Ann. 339 (2007), 485-544.

[25] G. Mingione. A. Zatorska-Goldstein and X. Zhong. Gradient regularity for elliptic equations in the Heisenberg group. Adv. Math. 222 (2009), 62-129.

[26] A. Nagel, E. M. Stein and S. Wainger. Balls and metrics defined by vector fields I. Basic properties. Acta Math. 155 (1985), 130-147.

[27] L. P. Rothschild and E. M. Stein. Hypoelliptic differential operators and nilpotent groups. Acta Math. 137 (1976), 247-320.

[28] J. Wang, P. Niu and X. Cui. $L^{p}$ estimates for weak solutions to nonlinear sub-elliptic systems related to Hörmander's vector fields. Kyushu J. Math. 63 (2009), 301-314.

[29] C. Xu. Regularity for quasi-linear second order subelliptic equations. Comm. Pure Appl. Math. 45 (1992), 77-96.

[30] C. Xu and C. Zuily. Higher interior regularity for quasilinear subelliptic systems. Calc. Var. Partial Differential Equations 5 (1997), 323-343.

\author{
Jialin Wang \\ Key Laboratory of Numerical Simulation Technology of Jiangxi Province \\ and \\ School of Mathematics and Computer Science \\ Gannan Normal University \\ Ganzhou 341000 \\ Jiangxi, P.R. China \\ (E-mail: jialinwang1025@hotmail.com) \\ Dongni Liao \\ School of Mathematics and Computer Science \\ Gannan Normal University \\ Ganzhou 341000 \\ Jiangxi, P.R. China \\ (E-mail:dongniliao@hotmail.com)
}

